\title{
Responsiveness of Instruments to Assess Disorders of Consciousness: A Literature Review
}

\author{
Emmah J. Doig ${ }^{1}$ and Amanda T. Lane-Brown ${ }^{2}$ \\ ${ }^{1}$ The University of Queensland, School of Health and Rehabilitation Sciences, Brisbane, Australia \\ ${ }^{2}$ Brain Injury Rehabilitation Unit, Liverpool Hospital \& Rehabilitation Studies Unit, University of Sydney, \\ Sydney, Australia
}

\begin{abstract}
Objectives: To summarise available evidence for responsiveness of six key assessments used with patients with disorders of consciousness: Coma Recovery Scale Revised (CRS-R), Disorders Of Consciousness Scale (DOCS), Sensory Modality Assessment and Rehabilitation Technique (SMART), Sensory Stimulation Assessment Measure (SSAM), Wessex Head Injury Matrix (WHIM), and the Western Neuro Sensory Stimulation Profile (WNSSP).

Method: A literature search of five electronic databases was conducted using a systematic search strategy. Relevant literature was evaluated and pertinent information extracted.

Results: Database searches using key terms initially yielded 132 articles. Following review for inclusion identified 24 articles. No studies were specifically designed to investigate responsiveness of any of the measures and therefore responsiveness data were either based on statistical significance of change post-treatment or descriptive analysis of change scores. The majority of studies identified used the CRS-R $(n=11)$, WHIM $(n=5)$ and WNSSP $(n=6)$ and have established responsiveness to change. There is some preliminary evidence for the responsiveness of the other measures, based on very few available studies: DOCS $(n=2)$, SMART $(n=1)$ or SSAM $(n=1)$.

Conclusion: Future studies should seek to include responsiveness analysis, particularly in relation to the DOCS, SMART and SSAM.
\end{abstract}

Keywords: outcome measures, consciousness, assessment, acquired brain injury

\section{Introduction}

In some instances, acquired brain injury $(\mathrm{ABI})$ can result in extended periods of reduced consciousness where individuals may be described as 'slow-to-recover'. According to the Royal College of Physicians (2003) guidelines, between 10 and $20 \%$ of people who experience moderate to severe brain injury may remain in either a vegetative state (VS) or minimally conscious state (MCS). Impaired awareness and responsiveness are characteristic of disordered consciousness and the stages of recovery of consciousness have been defined by consensus-based diagnostic criteria which differentiate coma, VS and MCS (Giacino et al., 1997, 2002).

Coma requires absence of eye opening, verbalisation or mouthing of words, response to commands and intentional movement. Emergence from coma to VS requires periods of eye opening and return of autonomic functions, but there is no evidence of the ability to follow commands, make intelligible verbal responses, make verbal or gestural attempts to communicate or exhibit localising or automatic motor responses (Giacino \& Kalmar, 
1997). Furthermore, VS has been defined as "no evidence of sustained, reproducible, purposeful or voluntary behavioural responses to visual, auditory, tactile or noxious stimuli' (The Multi-Society Task Force on Persistent Vegetative State, 1994, p. 1499). Minimally conscious state, as defined by the Aspen Workgroup on the Vegetative and MCS, is 'a condition of severely altered consciousness in which the person demonstrates minimal but definite behavioural evidence of self or environmental awareness' (Giacino et al., 1997, p. 84). One or more of the following behaviours needs to be observed and reproduced (at least inconsistently) to determine emergence from VS to MCS: comprehension of simple commands, manipulation of objects, gestural or verbal yes/no responses, intelligible verbalisations or stereotypical (not reflexive) movements (Giacino et al., 1997).

There are a number of behavioural assessments which are used by clinicians to assess consciousness. Behavioural assessments of consciousness involve observation of a range of behaviours associated with emergence of consciousness, such as arousal, responsiveness to structured and naturally occurring stimuli, communication and purposeful movement. Clinicians use such assessments to monitor changes in consciousness over time. Having a reliable and valid assessment measure is a necessity in order to ensure results are accurate. An additional principal element required to maximise utility is responsiveness. Responsiveness is the sensitivity of a measure to detect true or reliable changes occurring in the individual over time, above and beyond random fluctuations or error inherent in the test score. Responsiveness is essential for assessing the effectiveness of treatment and longitudinal change over time (Guyatt, Deyo, Charlson, Levine, \& Mitchell, 1989). If measures are sensitive and able to reliably detect change over time, they can help clinicians to determine readiness for rehabilitation in a timely manner, plan and implement appropriate treatments, reliably detect changes in response to treatment and justify treatment efforts and funding. Multiple methods have been suggested to determine responsiveness including paired $t$-test, receiver operating characteristic curve and effect size statistics (Kazis, Anderson, \& Meenan, 1989; Portney \& Watkins, 2000); however, there is no consensus on the most ideal method (Husted, Cook, Farewell, \& Gladman, 2000; Wright \& Young, 1997).

A recent systematic review by Seel and colleagues (2010) of behavioural assessment scales of disorders of consciousness resulted in evidencebased recommendations about their clinical use. The review examined the available evidence for content validity, reliability, ability to predict func- tional outcomes and diagnostic validity of assessments of disorders of consciousness, and was conducted by an expert panel of 14 members (Seel et al., 2010). The systematic review included 37 articles which represented 13 assessment scales for disorders of consciousness (Seel et al., 2010). The evidence was rated using several evidence-rating schemes, including: the Aspen Workgroup criteria for transitioning from VS to MCS and emerging from MCS (used to assess content validity), the American Academy of Neurology (AAN) four-tier level of evidence scheme (used to classify studies addressing diagnostic and prognostic validity) and the task force-developed three-tier evidence scheme (used to classify studies addressing reliability) (Seel et al., 2010). Clinical practice recommendations were based on the evidence and used a modified AAN strength of recommendation scheme to classify assessments as recommended for use with minor, moderate or major reservations, not recommended at this time, or not recommended (Seel et al., 2010). Ultimately, they recommended that the Coma Recovery Scale - Revised (CRS-R) may be used to assess disorders of consciousness with minor reservations and the Disorders Of Consciousness Scale (DOCS), Sensory Modality Assessment and Rehabilitation Technique (SMART), Sensory Stimulation Assessment Measure (SSAM), Wessex Head Injury Matrix (WHIM), and the Western Neuro Sensory Stimulation Profile (WNSSP) may be used with moderate reservations. The remaining seven assessment scales included were either recommended for use with major reservations, or were not recommended for use at this time or not recommended. Measures that were recommended for clinical use with minor or moderate reservations in the review by Seel and colleagues (2010) were included in the current review.

\section{Summary of Recommended Measures}

\section{Coma Recovery Scale - Revised}

The JFK Coma Recovery Scale - Revised (Giacino, Kalmar \& Whyte, 2004) is a clinicianadministered scale that assesses patients with disorders of consciousness, assists with differential diagnosis between coma, VS and MCS and can be used for treatment planning and monitoring. This revised scale was developed in line with the diagnostic recommendations of the Aspen Workgroup on MCS and was designed for patients with ABI with Ranchos Los Amigos Levels of Cognitive Functioning (RLAS) from Level I through to IV. The 23 items are divided into six subscales, each hierarchically organised from reflexive to cognitively mediated 
behaviours, including auditory, visual, motor, oromotor, communication and arousal processes. Scoring is based on the presence of a behavioural response to stimuli. Psychometric properties are sound; inter-rater reliability is high $(r=0.84)$, as is test-retest reliability ( $r=0.94)$ (Giacino et al., 2004). It has demonstrated concurrent validity with the Disability Rating Scale (DRS) (Rappaport, Hall, Hopkins, Belleza, \& Cope, 1982) and internal consistency is high ( $r=-0.90$ and Cronbach's $\alpha=0.83$, respectively) (Giacino et al., 2004).

\section{Disorders of Consciousness Scale (DOCS)}

The DOCS (Pape, Heinemann, Kelly, Hurder, \& Lundgren, 2005a) was designed to detect and distinguish changes in consciousness, providing a description of neuro-behavioural recovery. The DOCS includes 23 test stimuli organised into eight subscales: social knowledge, taste and swallowing, olfactory, proprioceptive and vestibular, auditory, visual, tactile and testing-readiness. A baseline observation is taken whereby a checklist is completed by the examiner after observing the person at rest. Testing stimuli are administered following baseline observation, where scores are allocated based on the best behavioural response to stimuli on a three-point scale $(0=$ no response, $1=$ generalised response, and $2=$ localised response). A higher score on the DOCS scale indicates a higher level of neuro-behavioural integrity. The DOCS demonstrates good internal consistency (Cronbach's $\alpha=$ 0.85 ) and construct validity was determined through Rasch modelling, which showed some evidence of unidimensional hierarchic interval characteristics (Pape et al., 2005a).

\section{Sensory Modality Assessment and Rehabilitation Technique (SMARD)}

The SMART was designed as an assessment and treatment tool to distinguish accurately patients in VS and MCS and then direct and evaluate rehabilitation efforts to facilitate recovery (Gill-Thwaites \& Munday, 2004). The SMART assesses eight modalities including five sensory modalities (visual, auditory, tactile, olfactory and gustatory) and three other modalities comprising motor function, functional communication and wakefulness/arousal. Each modality is rated on a five-level hierarchical scale (level $1=$ no response; level 2 = reflexive; level 3 =withdrawal; level 4 $=$ localization; and level $5=$ discrimination). The five levels relate directly to RLAS Levels I-IV. If a patient is observed to perform at level 5 in any of the seven modalities, such behaviour is incompatible with a diagnosis of VS. The total score can range from 7 to 35; however, in a clinical context, SMART scores are not summed, rather levels of function in modalities are reported. The formal component of the SMART assessment is conducted by SMART-trained and accredited assessors and comprises a sensory assessment and behavioural observation assessment. The informal component of the SMART assessment consists of information gathered from family and carers regarding observed behaviours and previous interests and preferences. Psychometric properties are sound: inter-rater reliability is excellent $(r=0.96)$, as is test-retest reliability (ICC of 0.97 ), with moderate concurrent validity with the WNSSP ( $r=$ 0.70) (Gill-Thwaites \& Munday, 2004).

\section{Sensory Stimulation Assessment Measure (SSAM)}

The SSAM (Rader \& Ellis, 1994) was developed to distinguish changes in consciousness, to provide an accurate, replicable assessment of responsiveness in people with ABI between RLAS Levels II through V, and to assist treatment planning. Stimuli are presented to five senses and the best response is measured using the sensory stimulation response scale (SSRS) within three categories: eye opening, motor and vocalisation. Each category has six response choices, ranging from 'no response' to 'able to follow commands or communicate ideas'. Scores are summed and range from 3 to 36 , giving a total response score. Test-retest reliability is excellent $(r=0.93)$, inter-rater reliability is high $(r=$ $0.89)$, and the SSAM has demonstrated moderate concurrent validity with the GCS $(r=0.70)$ and the DRS ( $r=0.61)$ (Rader, Alston, \& Ellis, 1989; Rader \& Ellis, 1994).

\section{Wessex Head Injury Matrix (WHIM)}

The WHIM was developed by a multidisciplinary team (Shiel, Wilson, McLellan, Horn, \& Watson, 2000a) following observations of 88 patients recovering from severe traumatic brain injury (TBI). It is designed to detect subtle changes in the early recovery of cognition, social behaviour, communication and attention, and to identify a sequence of recovery from coma to emergence from posttraumatic amnesia (PTA). The WHIM contains 62 items which are hierarchically organised, with items rank ordered in terms of their sequence of recovery. Spontaneous behaviours, responses to presentation of standardised stimuli and responses to naturally occurring stimuli are examined. The WHIM score is the rank number of the highest behaviour successfully passed in the sequence of 62 behaviours. Psychometric properties include good 
inter-rater reliability $(k=0.86)$ and test-retest reliability $(k=0.74)$ (Shiel et al., 2000a). It has demonstrated strong concurrent validity with the Glasgow Coma Scale (GCS) (initial WHIM $r=$ 0.83 and final WHIM $r=0.95$ ) (Majerus \& Van de Linden, 2000).

\section{Western Neuro Sensory Stimulation Profile (WNSSP)}

The WNSSP was developed for use with people with TBI who are classified as RLAS Level II through V, which includes people in VS and MCS (Ansell \& Keenan, 1989). The tool was designed to evaluate cognitive status, monitor progress and predict improvements in patients who are in VS and MCS. The WNSSP consists of 33 items divided into six subscales (arousal/attention, auditory comprehension, visual comprehension, visual tracking, object manipulation and expressive communication). Total scores range from 0 to 113 , with higher scores indicating greater abilities. Psychometric properties include concurrent validity with RLAS $(r=0.73)$ and excellent internal consistency (Cronbach's $\alpha=0.95$ ) (Ansell \& Keenan, 1989).

\section{Objectives}

The review by Seel and colleagues (2010) provided a highly valuable examination of the available evidence for the reliability, diagnostic validity (including criterion validity) and prognostic validity of different assessment scales for disorders of consciousness. Their review did not examine responsiveness of the different scales. Thus, to the best of the authors' knowledge there is no known examination of the evidence available about the ability of the scales to detect meaningful change. This knowledge has obvious clinical utility. It is essential that clinicians are able to determine whether their patients are getting better (or worse) through accurate, objective means. To address this gap in the literature, and complement the findings of Seel and colleagues (2010), the present review examines the literature to explore the state of the evidence in relation to the responsiveness of the CRSR, DOCS, SMART, SSAM, WHIM, and WNSSP.

\section{Methods}

\section{Search Strategy for Identifying Literature}

Relevant studies were identified from the following five electronic bibliographic databases: Medline, PsycINFO, Cumulative Index to Nursing and Allied Health Literature (CINAHL) and Cochrane Database of Systematic Reviews (DSR) and Cochrane Central Register of Controlled Tri- als (CCTR). A search was conducted on $16 \mathrm{Au}$ gust 2012. Search strategies used keywords of the six full assessment titles: Coma Recovery Scale Revised, Disorders of Consciousness Scale, Sensory Modality Assessment and Rehabilitation Technique, Sensory Stimulation Assessment Measure, Wessex Head Injury Matrix, and Western Neuro Sensory Stimulation Profile. Other resources searched were reference lists of included trials and the authors' personal collections.

\section{Inclusion Criteria}

Criteria for including trials were as follows: (1) human adult participants; (2) reported in the English language; (3) reported on the use of at least one of the disorders of consciousness measures (CRSR, DOCS, SMART, SSAM, WHIM, WNSSP); (4) took repeated measures of participants using the MCS tool on at least two occasions at different points in time; (5) reported total scores on at least two different time points and/or reported on change in scores between at least two different time points; (6) reported or described the participants as involved in a treatment programme during the assessment period, even if the stated purpose of the study was not to test the effectiveness of a treatment. For example, studies were included that did not specifically state that the aim was to test the effectiveness of a treatment but reported outcomes of participants over time where the participants were, for instance, inpatients in a brain injury rehabilitation setting. These studies were included despite the lack of description of specific details of treatment (i.e., nature, intensity, duration) because it was assumed that the patients in these studies were receiving some treatment or management of their condition during the assessment period, given their presence in those settings.

\section{Procedure}

Results of searches were tracked through EndNote, a reference-management system. Titles and abstracts were reviewed against inclusion criteria by E.D. If the abstracts and titles did not provide adequate information to evaluate criteria for inclusion, a full-text version of the article was reviewed by E.D. to confirm inclusion or exclusion. Templates were developed and used by both authors to extract relevant information from each included paper about responsiveness, including assessment time-points, statistical methods used to determine change and findings. Data about participants (injury severity and demographic information) as well as nature, intensity and length of treatment interventions were also extracted to enable the authors to consider potential reasons for any variations in 
findings between studies. Data about change as measured by any other assessments of consciousness that were administered at the same time-points as the six assessments that were the subject of this review were also extracted. These data were included to compare the responsiveness of the six measures relative to other measures used. Both authors reviewed and extracted data for all included papers.

Cohen's $d$ measures the size of the treatment effect. It is important to determine effect size, in addition to statistical significance, because statistical significance does not necessarily imply that meaningful change has occurred. For example, a statistically significant change may be observed solely because of a large sample size, regardless of whether this change is clinically meaningful (Olejnik \& Algina, 2000). Thus, calculating the effect size is a way to demonstrate that meaningfully significant change has occurred. Cohen's $d$ was calculated using the difference between two means divided by the pooled standard deviation weighted by sample size (Cohen, 1988; Zakzanis, 2001). According to Cohen, a $d$ value of 0.8 or higher represents a large effect; 0.5 , a moderate effect; and 0.2, a small effect. Meta-analysis was not conducted due to the heterogeneity of the studies identified.

\section{Results}

Results of all searches yielded 132 references. Of these, 40 trials met the inclusion criteria and full text articles were reviewed. Following review of the full text articles, 25 were included in the final review: these include articles related to the CRS-R $(n=11)$, DOCS $(n=2), \operatorname{SSAM}(n=1)$, WHIM $(n=5)$ and WNSSP $(n=6)$. Of the six relating to the WNSSP, one of these concurrently reported on the SMART. Data were extracted from included papers and are presented in Tables $1-4$. Cohen's $d$ is reported in the findings column of the summary tables, where it was possible to calculate it.

In relation to the CRS-R (Table 1 ), only 3 of the 11 papers $(27.3 \%)$ utilised statistical analysis methods to measure change in the CRS-R scores. Data were often presented solely via description or graphical representation. The vast majority (10 of the 11 papers, $91 \%$ ) reported a change in CRS-R scores following treatment in at least some study participants. Of the three studies reporting results of statistical analysis, two of the three demonstrated a significant change in CRS-R score following treatment. It was possible to calculate the effect size of the change in scores in one paper (DeFina et al., 2010), where a large effect size ( $d=2.01-5.11$ for different groups) was observed. This provides evidence that the CRS-R is responsive to change. Additionally, some studies provided information about changes on the CRS$\mathrm{R}$ simultaneously with changes on other outcome measures examining disorders of consciousness (i.e., concurrent validity). In these studies it was observed that the CRS-R and DRS provided similar patterns of responsiveness to change in participants. However, the level of agreement between the CRS-R and Coma/Near Coma Scale (C/NCS) (Rappaport, Dougherty, \& Kelting, 1992) varied. Primarily, there was a consistent pattern of results for both the CRS-R and C/NCS when correlated with the level of drug concentration in two studies (McMahon, Vargus-Adams, Michaud, \& Bean, 2009; Vargus-Adams, McMahon, Michaud, Bean, $\&$ Vinks, 2010). This provides preliminary evidence for concurrent validity; however, future studies would benefit from measuring this directly by correlating the scores of the two measures.

The six papers measuring change using the WNSSP, one of which concurrently measured change using the SMART, are outlined in Table 2 . Of these studies, $3 / 6$ used statistical analysis to determine significance of change over time, one of which related to the SMART. It was possible to calculate effect size for four papers (Lammi, Smith, Tate, \& Taylor, 2005; Patrick et al., 2009; Smith, Taylor, Lammi, \& Tate, 2001; Wilson \& Gill-Thwaites, 2000), mostly indicating effect sizes over 0.8 . These data support the responsiveness of the WNSSP (Lammi et al., 2005; Patrick et al., 2009) and SMART (Wilson \& GillThwaites, 2000). Two studies concurrently reported on change on other consciousness scales indicating agreement between the WNSSP and RLAS, Rader's scale and GCS (Hall, MacDonald, \& Young, 1992) and between the WNSSP and the Post Acute Level of Consciousness Scale (PALOCS) (Eilander et al., 2009). However, sample sizes were small $(n=6$ and $n=3)$ and agreement was not determined using statistical analysis, rather descriptive analysis.

The findings of the five studies measuring change using the WHIM are detailed in Table 3. Three of the five papers used statistical analysis to determine the significance of change and Cohen's $d$ was calculated for three papers (Wilson, Coleman, \& Pickard, 2008; Wilson, Elder, McCrudden, \& Caldwell, 2009; Wilson, Harpur, Watson, \& Morrow, 2003). Effect sizes were in excess of 0.8 for two of the studies (Wilson et al., 2008, 2009) with moderate effect sizes for a smaller sample of seven participants (Wilson et al., 2003). This same study by Wilson and colleagues (2003) compared improvement according to the GCS 
TABLE 1

Summary of studies that have measured change using CRS-R

\begin{tabular}{|c|c|c|c|c|c|c|}
\hline $\begin{array}{l}\text { Study (author, } \\
\text { date, country } \\
\text { of lead author) }\end{array}$ & $\begin{array}{l}\text { No. of participants/ } \\
\text { diagnosis/injury severity } \\
\text { on admission to study } \\
\text { (ranges, means/VS/ } \\
\text { MCS/RLAS/GCS)/ Time } \\
\text { post-injury/ Sex /Age } \\
\text { (range, mean) }\end{array}$ & $\begin{array}{l}\text { Treatment (including } \\
\text { duration and intensity of } \\
\text { treatment) }\end{array}$ & $\begin{array}{l}\text { Statistical } \\
\text { method to } \\
\text { determine } \\
\text { change on } \\
\text { CRS-R }\end{array}$ & $\begin{array}{l}\text { Assessment time points for } \\
\text { CRS-R }\end{array}$ & $\begin{array}{l}\text { Other outcome } \\
\text { measures at } \\
\text { concurrent } \\
\text { time-points }\end{array}$ & Findings \\
\hline $\begin{array}{l}\text { Bekinschtein } \\
\quad \text { et al., } 2005 \\
\text { Argentina }\end{array}$ & 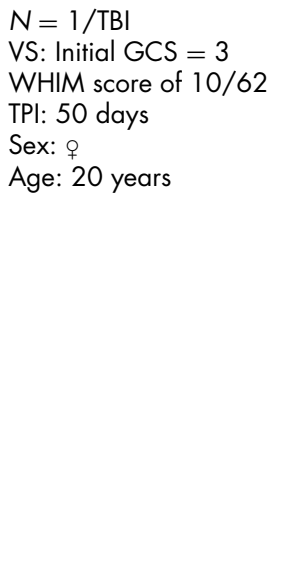 & $\begin{array}{l}\text { 1. Post-injury days } 50- \\
113=\text { integrative } \\
\text { multisensory programme } \\
\text { for two 2-hour sessions } \\
\text { daily. } \\
\text { 2. Days } 114-235= \\
\text { cognitive rehabilitation } \\
\text { programme, individual } \\
\text { and group sessions. } \\
\text { Individual sessions ( } 30 \text { min } \\
\text { daily) focused around } \\
\text { cognitive goals and } \\
\text { cognitive behaviour } \\
\text { therapy. Group weekly } \\
\text { sessions included } \\
\text { education about ABI. }\end{array}$ & Descriptive & $\begin{array}{l}6 \text { times between days } 66 \\
\text { and } 122 \text { post-injury } \\
\text { (every } 1-2 \text { weeks) }\end{array}$ & $\begin{array}{l}\text { WHIM (5 times } \\
\text { between } \\
\text { day } 51 \text { and } \\
235 \text { days } \\
\text { post-injury). } \\
\text { DRS } \\
\text { (concurrently } \\
\text { with CRS-R) }\end{array}$ & $\begin{array}{l}\text { CRS-R between days } 66 \\
\text { and } 108 \text { post-injury - } \\
\text { values ranged between } \\
17 / 25 \text { and } 19 / 25 \text {. By } \\
\text { day } 122 \text { this increased } \\
\text { to } 24 \text {. } \\
\text { Total agreement between } \\
\text { the DRS and CRS-R was } \\
\text { observed. However, the } \\
\text { DRS demonstrated a } \\
\text { ceiling effect, that was } \\
\text { not present in the CRS-R. } \\
\text { WHIM score at day } 51 \\
\text { post- injury was } 10 / 62 \text {; } \\
\text { at day } 81 \text { was } 24 / 62 ; \\
\text { at day } 112 \text { was } 41 / 62 \text {. } \\
\text { CRS allowed mapping of } \\
\text { both MCS and high MCS }\end{array}$ \\
\hline $\begin{array}{l}\text { DeFina et al., } \\
2010 \\
\text { USA }\end{array}$ & $\begin{array}{l}N=41 / \mathrm{TBI}=21 \\
\text { non- } \mathrm{TBI}=20 \\
\mathrm{VS}=32(\mathrm{TBI}=4 \\
\text { non } \mathrm{TBI}=18)\end{array}$ & $\begin{array}{l}\text { Advanced Care Protocol } \\
\text { (ACP): }\end{array}$ & $\begin{array}{l}\text { Wilcoxon } \\
\text { Z } \\
\text { statistic }\end{array}$ & $\begin{array}{l}\text { Baseline measures within } \\
48 \mathrm{~h} \text { of admission (pre) } \\
\text { and after the ACP (post) }\end{array}$ & $\begin{array}{l}\text { DRS } \\
\text { GCS }\end{array}$ & $\begin{array}{l}\text { In VS-TBI group }(n=14): \\
\text { Pre: Mean }=6.43, S D= \\
\quad 3.01\end{array}$ \\
\hline
\end{tabular}


TABLE 1

Continued.

\begin{tabular}{|c|c|c|c|c|c|c|}
\hline $\begin{array}{l}\text { Study (author, } \\
\text { date, country } \\
\text { of lead author) }\end{array}$ & $\begin{array}{l}\text { No. of participants/ } \\
\text { diagnosis/injury severity } \\
\text { on admission to study } \\
\text { (ranges, means/VS/ } \\
\text { MCS/RLAS/GCS)/ Time } \\
\text { post-injury/ Sex /Age } \\
\text { (range, mean) }\end{array}$ & $\begin{array}{l}\text { Treatment (including } \\
\text { duration and intensity of } \\
\text { treatment) }\end{array}$ & $\begin{array}{l}\text { Statistical } \\
\text { method to } \\
\text { determine } \\
\text { change on } \\
\text { CRS-R }\end{array}$ & $\begin{array}{l}\text { Assessment time points for } \\
\text { CRS-R }\end{array}$ & $\begin{array}{l}\text { Other outcome } \\
\text { measures at } \\
\text { concurrent } \\
\text { time-points }\end{array}$ & Findings \\
\hline & $\begin{array}{l}M C S=9(T B I=7, \\
\text { non-TBI = 2) } \\
\text { TPI: For } 39 \text { participants TPI } \\
\text { was between } 1 \text { month } \\
\text { and } 12 \text { months. For } 2 \\
\text { participants, TPI was } \\
\text { over } 1 \text { year. Mean TPI = } \\
6 \text { months } \\
\text { Sex: } \sigma^{7}=29,9=12 \\
\text { Age: } V S-T B I: \text { Mean }= \\
26.93, S D=10.21 \\
\text { Age: } V S-\text { non-TBI: } \\
M=46.89, S D=18.28 \\
\text { Age: } M C S-T B I: \\
M=26.29, S D=14.37 \\
\text { Age: } M C S-n o n-T B I: \\
M=53.33, S D=9.90\end{array}$ & $\begin{array}{l}\text { Standard care (nursing, } \\
\text { physical therapy, } \\
\text { occupational therapy, } \\
\text { speech pathology for } 3 \\
\text { hours per weekday, } \\
\text { modified on weekends) } \\
\text { PLUS sequential, } \\
\text { cumulative introduction } \\
\text { of 3-phase treatment, } \\
\text { introduced at rate of } 1 \\
\text { phase/fortnight. Final } \\
\text { treatment phase } \\
\text { maintained until } \\
\text { discharge (on average } \\
\text { after } 12 \text { weeks). } \\
\text { Phase 1: Targeted } \\
\text { pharmacological } \\
\text { treatment customised to } \\
\text { be clinically appropriate } \\
\text { for each patient to } \\
\text { optimise or potentiate } \\
\text { neurotransmitter function } \\
\text { (e.g., naltrexone, } \\
\text { amantadine, } \\
\text { levo/carbidopa). } \\
\text { Phase } 2: \text { Median nerve } \\
\text { stimulation administered } \\
\text { with bilateral external } \\
\text { stimulation applied } \\
\text { randomly to left or right } \\
\text { arm for } 8 \text { hours per day, } \\
7 \text { days/week. }\end{array}$ & & & & 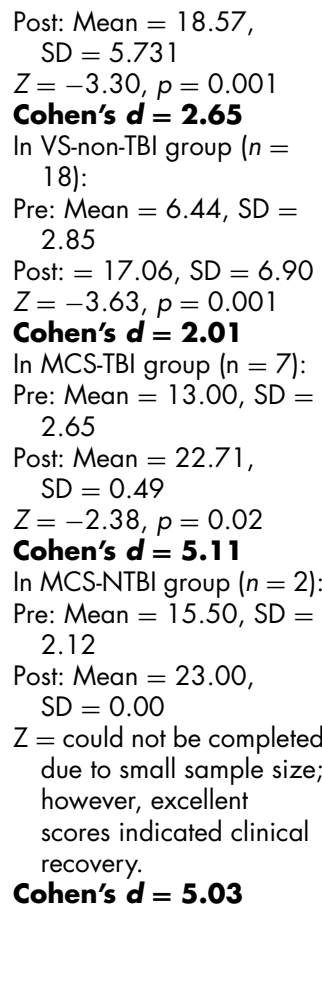 \\
\hline
\end{tabular}


TABLE 1

Continued.

\begin{tabular}{|c|c|c|c|c|c|c|}
\hline $\begin{array}{l}\text { Study (author, } \\
\text { date, country } \\
\text { of lead author) }\end{array}$ & $\begin{array}{l}\text { No. of participants/ } \\
\text { diagnosis/injury severity } \\
\text { on admission to study } \\
\text { (ranges, means/VS/ } \\
\text { MCS/RLAS/GCS)/ Time } \\
\text { post-injury/ Sex /Age } \\
\text { (range, mean) }\end{array}$ & $\begin{array}{l}\text { Treatment (including } \\
\text { duration and intensity of } \\
\text { treatment) }\end{array}$ & $\begin{array}{l}\text { Statistical } \\
\text { method to } \\
\text { determine } \\
\text { change on } \\
\text { CRS-R }\end{array}$ & $\begin{array}{l}\text { Assessment time points for } \\
\text { CRS-R }\end{array}$ & $\begin{array}{l}\text { Other outcome } \\
\text { measures at } \\
\text { concurrent } \\
\text { time-points }\end{array}$ & Findings \\
\hline & & $\begin{array}{l}\text { Phase 3: Nutraceutical } \\
\text { treatment - a customised } \\
\text { combination of nutrients, } \\
\text { vitamins and } \\
\text { antioxidants. }\end{array}$ & & & & $\begin{array}{l}\text { GCS and DRS showed } \\
\text { statistically significant } \\
\text { improvement for all } 4 \\
\text { groups concurrently with } \\
\text { CRS-R }\end{array}$ \\
\hline $\begin{array}{l}\text { Katz, Polyak, } \\
\text { Coughlan, } \\
\text { Nicols, \& } \\
\text { Roche, } \\
2009 \\
\text { USA }\end{array}$ & $\begin{array}{l}N=36 / A B I(T B I=22, \\
\text { non-TBI=14) } \\
\text { VS }=11 \\
M C S=25 \\
\text { TPI: } M=35 \text { days, } S D= \\
25.9 \text { days } \\
\text { Sex: } 0^{7}=22, q=14 \\
\text { Age: } M=38 \text { years (SD = } \\
21) \\
\text { TBI: } M=29 \text { years (SD = } \\
\text { 18) } \\
\text { Non-TBI: } M=50 \text { years } \\
\text { (SD }=18 \text { ) }\end{array}$ & $\begin{array}{l}\text { Specialist inpatient brain } \\
\text { injury rehabilitation } \\
\text { programme for patients } \\
\text { with prolonged } \\
\text { disorders of } \\
\text { consciousness. } \\
\text { Treatment: Physical, } \\
\text { occupational and } \\
\text { speech therapies } \\
\text { totalling at least } 3 \text { hours } \\
\text { per day. Treated with a } \\
\text { dopamine agonist or } \\
\text { stimulant medication at } \\
\text { some point during } \\
\text { admission. }\end{array}$ & $\begin{array}{l}\text { Does not } \\
\text { specifi- } \\
\text { cally } \\
\text { state } \\
\text { analysis } \\
\text { used for } \\
\text { CRS-R }\end{array}$ & $\begin{array}{l}\text { At least } 1-2 \text { times per } \\
\text { week while in VS or } \\
\text { MCS }\end{array}$ & $\begin{array}{l}\text { DRS } \\
\text { (followed up } \\
\text { from } \\
\text { discharge } \\
\text { and yearly } \\
\text { at } 1-4 \text { years } \\
\text { post-injury) }\end{array}$ & $\begin{array}{l}\text { CRS-R used to measure } \\
\text { changes from VS to MCS } \\
\text { and emergence from } \\
\text { MCS. Findings for CRS-R } \\
\text { not individually } \\
\text { specified. } \\
\text { DRS at } 1 \text { year post-injury: } \\
\text { Mean = 9.8, SD = } \\
6.79 \text {. When participants } \\
\text { were followed up } \\
\text { between } 1 \text { and } 4 \text { years, } \\
\text { follow-up DRS showed } \\
\text { that } 27 \% \text { improved to a } \\
\text { score of } 3 \text { or less } \\
\text { (partial, mild or no } \\
\text { disability), } 41 \% \text { had } \\
\text { moderate to moderately } \\
\text { severe disability, and } \\
32 \% \text { scored between } 12 \\
\text { and } 21 \text { (severe to } \\
\text { extremely severe } \\
\text { disability). }\end{array}$ \\
\hline
\end{tabular}


TABLE 1

Continued.

\begin{tabular}{|c|c|c|c|c|c|c|}
\hline $\begin{array}{l}\text { Study (author, } \\
\text { date, country } \\
\text { of lead author) }\end{array}$ & $\begin{array}{l}\text { No. of participants/ } \\
\text { diagnosis/injury severity } \\
\text { on admission to study } \\
\text { (ranges, means/VS/ } \\
\text { MCS/RLAS/GCS)/ Time } \\
\text { post-injury/ Sex /Age } \\
\text { (range, mean) }\end{array}$ & $\begin{array}{l}\text { Treatment (including } \\
\text { duration and intensity of } \\
\text { treatment) }\end{array}$ & $\begin{array}{l}\text { Statistical } \\
\text { method to } \\
\text { determine } \\
\text { change on } \\
\text { CRS-R }\end{array}$ & $\begin{array}{l}\text { Assessment time points for } \\
\text { CRS-R }\end{array}$ & $\begin{array}{l}\text { Other outcome } \\
\text { measures at } \\
\text { concurrent } \\
\text { time-points }\end{array}$ & Findings \\
\hline $\begin{array}{l}\text { Lancioni et al., } \\
2011 \\
\text { Italy }\end{array}$ & 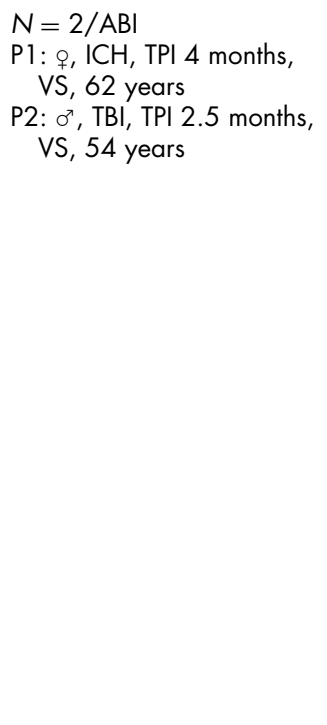 & $\begin{array}{l}\text { Micro-switch activation via } \\
\text { eyelid closure in } \\
\text { (A) Baseline phases - did } \\
\text { not produce any } \\
\text { stimulation } \\
\text { (B) Intervention phases - } \\
\text { was followed by } 10 \\
\text { seconds of preferred } \\
\text { music } \\
\text { (C) Control phases - } \\
\text { preferred music was } \\
\text { played continuously } \\
\text { (micro-switch responses } \\
\text { were recorded) } \\
\text { Between } 2 \text { and } 11 \\
\text { sessions occurred each } \\
\text { day for } 5 \text { minutes per } \\
\text { session. One participant } \\
\text { had a total of } 415 \\
\text { sessions (baseline, } \\
\text { treatment and control), } \\
\text { and the other had } 206 \\
\text { sessions. }\end{array}$ & $\begin{array}{c}\text { Descriptive } \\
\text { analysis } \\
\text { of micro- } \\
\text { switch } \\
\text { activa- } \\
\text { tion } \\
\text { during } \\
\text { A, B } \\
\text { and C } \\
\text { phases }\end{array}$ & $\begin{array}{l}6 \text { times across the } \\
\text { intervention and control } \\
\text { phases }\end{array}$ & $\begin{array}{l}\text { (Individual } \\
\text { response) } \\
\text { Activation of } \\
\text { the optic } \\
\text { micro-switch } \\
\text { controlled } \\
\text { by eyelid } \\
\text { closure } \\
\text { which } \\
\text { produced } \\
\text { music } \\
\text { stimulation } \\
\text { during B } \\
\text { phases }\end{array}$ & $\begin{array}{l}\text { Female participant scored } \\
6 \text { on CRS-R with } \\
\text { exception of assessment } \\
\text { occasion } 5 \text { where score } \\
\text { was } 7 . \\
\text { Male participant scored } 5 \\
\text { on CRS-R on all } 6 \\
\text { assessment occasions. } \\
\text { Learning data - increased } \\
\text { responding (activation of } \\
\text { micro-switch) during B } \\
\text { phases. }\end{array}$ \\
\hline
\end{tabular}


TABLE 1

Continued.

\begin{tabular}{|c|c|c|c|c|c|c|}
\hline $\begin{array}{l}\text { Study (author, } \\
\text { date, country } \\
\text { of lead author) }\end{array}$ & $\begin{array}{l}\text { No. of participants/ } \\
\text { diagnosis/injury severity } \\
\text { on admission to study } \\
\text { (ranges, means/VS/ } \\
\text { MCS/RLAS/GCS)/ Time } \\
\text { post-injury/ Sex /Age } \\
\text { (range, mean) }\end{array}$ & $\begin{array}{l}\text { Treatment (including } \\
\text { duration and intensity of } \\
\text { treatment) }\end{array}$ & $\begin{array}{l}\text { Statistical } \\
\text { method to } \\
\text { determine } \\
\text { change on } \\
\text { CRS-R }\end{array}$ & $\begin{array}{l}\text { Assessment time points for } \\
\text { CRS-R }\end{array}$ & $\begin{array}{l}\text { Other outcome } \\
\text { measures at } \\
\text { concurrent } \\
\text { time-points }\end{array}$ & Findings \\
\hline $\begin{array}{l}\text { McMahon, } \\
\text { Vargus- } \\
\text { Adams, } \\
\text { Michaud, \& } \\
\text { Bean, 2009 } \\
\text { USA }\end{array}$ & $\begin{array}{l}N=7 / \mathrm{ABI}(\mathrm{TBI}=5, \\
\text { anoxia }=1, \mathrm{CVA}=1) \\
(5 \text { completed study) } \\
\text { MCS }=2, \mathrm{VS}=5 \\
\mathrm{GCS} \text { range }=3-6, M=4, \\
\mathrm{CNCS}>2 \\
\text { TPI: } 4-12 \text { weeks }(M=6.7 \\
\text { weeks) } \\
\text { Sex: } 0^{7}=6, \uparrow=1 \\
\text { Age: range }=6-18 \text { years, } \\
M= \\
12.7\end{array}$ & $\begin{array}{l}\text { Each participant was } \\
\text { randomised to receive } \\
\text { treatment ( } 3 \text { weeks of } \\
\text { amantadine) first or } \\
\text { placebo ( } 3 \text { weeks } \\
\text { placebo) first, followed } \\
\text { by } 1 \text { week washout and } \\
\text { alternate condition ( } 3 \\
\text { weeks placebo or } 3 \\
\text { weeks amantadine) } \\
\text { followed by washout. } \\
\text { Treatment ( } 3 \text { weeks } \\
\text { amantadine): } \\
\text { Week } 1=\text { amantadine, } 4 \\
\text { mg/kg/day divided into } \\
2 \text { equal doses } \\
\text { Weeks } 2 \& 3= \\
\text { amantadine, } 6 \\
\text { mg/ } 6 \text { /day divided into } \\
2 \text { equal doses } \\
\text { Placebo: } 3 \text {-week period } \\
\text { when placebo } \\
\text { administered }\end{array}$ & $\begin{array}{l}\text { Weekly av- } \\
\text { erages } \\
\text { calcu- } \\
\text { lated, } \\
\text { plotted } \\
\text { and a } \\
\text { single } \\
\text { best-fit } \\
\text { straight } \\
\text { line } \\
\text { deter- } \\
\text { mined. } \\
\text { Recov- } \\
\text { ery } \\
\text { slopes } \\
\text { during } \\
\text { treat- } \\
\text { ment } \\
\text { and } \\
\text { placebo } \\
\text { phases } \\
\text { com- } \\
\text { pared } \\
\text { using } \\
\text { paired } \\
\text { ftests }\end{array}$ & $\begin{array}{l}\text { Baseline, } 3 \text { times / week } \\
\text { (unless discharged home } \\
\text { - then } 2 \text { times/week) }\end{array}$ & $\begin{array}{l}\text { C/NCS } \\
\text { (3 times / } \\
\text { week), } \\
\text { clinical } \\
\text { assessment }\end{array}$ & 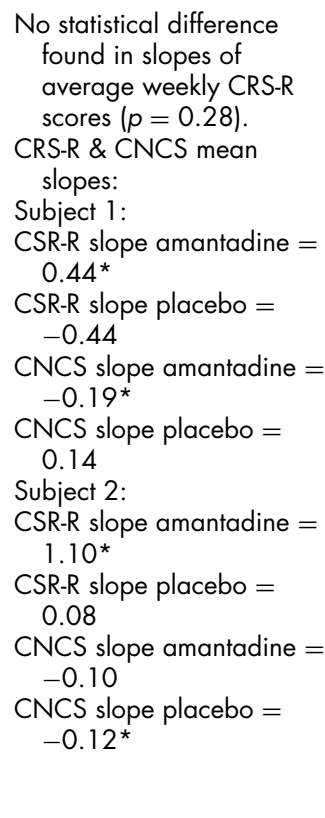 \\
\hline
\end{tabular}


TABLE 1

Continued.

\begin{tabular}{|c|c|c|c|c|c|c|}
\hline $\begin{array}{l}\text { Study (author, } \\
\text { date, country } \\
\text { of lead author) }\end{array}$ & $\begin{array}{l}\text { No. of participants/ } \\
\text { diagnosis/injury severity } \\
\text { on admission to study } \\
\text { (ranges, means/VS/ } \\
\text { MCS/RLAS/GCS)/ Time } \\
\text { post-injury/ Sex /Age } \\
\text { (range, mean) }\end{array}$ & $\begin{array}{l}\text { Treatment (including } \\
\text { duration and intensity of } \\
\text { treatment) }\end{array}$ & $\begin{array}{l}\text { Statistical } \\
\text { method to } \\
\text { determine } \\
\text { change on } \\
\text { CRS-R }\end{array}$ & $\begin{array}{l}\text { Assessment time points for } \\
\text { CRS-R }\end{array}$ & $\begin{array}{l}\text { Other outcome } \\
\text { measures at } \\
\text { concurrent } \\
\text { time-points }\end{array}$ & Findings \\
\hline & & & & & & $\begin{array}{l}\text { Subject } 4: \\
\text { CSR-R slope amantadine = } \\
0.42^{*} \\
\text { CSR-R slope placebo }= \\
-0.39 \\
\text { CNCS slope amantadine }= \\
0.02 \\
\text { CNCS slope placebo }= \\
-0.01^{*} \\
\text { Subject } 5: \\
\text { CSR-R slope amantadine = } \\
-0.17 \\
\text { CSR-R slope placebo }= \\
1.21^{*} \\
\text { CNCS slope amantadine = } \\
-0.08 \\
\text { CNCS slope placebo }= \\
-0.35^{*} \\
\text { Subject } 7: \\
\text { CSR-R slope amantadine }= \\
3.23^{*} \\
\text { CSR-R slope placebo }= \\
0.41 \\
\text { CNCS slope amantadine }= \\
-0.38^{*} \\
\text { CNCS slope placebo }= \\
-0.08 \\
\text { * more favourable slope }\end{array}$ \\
\hline
\end{tabular}


TABLE 1

Continued.

\begin{tabular}{|c|c|c|c|c|c|c|}
\hline $\begin{array}{l}\text { Study (author, } \\
\text { date, country } \\
\text { of lead author) }\end{array}$ & $\begin{array}{l}\text { No. of participants/ } \\
\text { diagnosis/iniury severity } \\
\text { on admission to study } \\
\text { (ranges, means/VS/ } \\
\text { MCS/RLAS/GCS)/ Time } \\
\text { post-injury/ Sex /Age } \\
\text { (range, mean) }\end{array}$ & $\begin{array}{l}\text { Treatment (including } \\
\text { duration and intensity of } \\
\text { treatment) }\end{array}$ & $\begin{array}{l}\text { Statistical } \\
\text { method to } \\
\text { determine } \\
\text { change on } \\
\text { CRS-R }\end{array}$ & $\begin{array}{l}\text { Assessment time points for } \\
\text { CRS-R }\end{array}$ & $\begin{array}{l}\text { Other outcome } \\
\text { measures at } \\
\text { concurrent } \\
\text { time-points }\end{array}$ & Findings \\
\hline $\begin{array}{l}\text { Noé et al., } \\
2012 \text { Spain } \\
\text { NOTE: } \\
\text { Used } \\
\text { Spanish } \\
\text { version of } \\
\text { CRS-R }\end{array}$ & $\begin{array}{l}\mathrm{N}=32 / \mathrm{ABI} \\
(\mathrm{TBI}=15, \mathrm{CVA}=12, \\
\text { Hypoxic-ischaemic }=5 \text { ) } \\
\text { VS or } \mathrm{MCS}, \mathrm{RLAS}=3 \text { or } \\
\text { lower } \\
\text { TPI: } 38-360 \text { days, } M= \\
144.9, \mathrm{SD}=81.6 \\
\text { Sex: } 0^{7}=22,0=10 \\
\text { Age: range }=16-64 \\
\text { years, } \\
M=39.9, \mathrm{SD}=13.9\end{array}$ & $\begin{array}{l}\text { Integrative multisensory } \\
\text { programme comprising } \\
\text { daily physical } \\
\text { rehabilitation } \\
\text { procedures and } \\
\text { multimodal sensory } \\
\text { stimulation }\end{array}$ & $\begin{array}{l}\text { No direct } \\
\text { analysis } \\
\text { of pre- } \\
\text { and } \\
\text { post- } \\
\text { treatment } \\
\text { change } \\
\text { in CRS-R } \\
\text { scores }\end{array}$ & $\begin{array}{l}\text { Baseline (before inclusion), } \\
\text { then monthly for at least } \\
6 \text { months of follow-up or } \\
\text { until emergence from } \\
\text { MCS }\end{array}$ & & $\begin{array}{l}\text { 'Patients' individual } \\
\text { trajectory of change } \\
\text {.. from baseline to the } \\
\text { moment of emerging } \\
\text { from MCS was not } \\
\text { uniform, with some } \\
\text { patients showing a slow } \\
\text { and progressive } \\
\text { increase, and others an } \\
\text { acute change' }\end{array}$ \\
\hline $\begin{array}{l}\text { Piccione et al., } \\
2011 \\
\text { Italy }\end{array}$ & $\begin{array}{l}N=1 / C V A \\
\text { MCS, GOS }=2, \text { CRS-R }= \\
14 \\
\text { TPI: } 5 \text { years } \\
\text { Sex: } \sigma^{7} \\
\text { Age: } 70 \text { years }\end{array}$ & $\begin{array}{l}\text { Three sessions with } 3 \text { days } \\
\text { between each session } \\
\text { (ABA design) } \\
\text { - Session } 1 \text { (Treatment) } \\
\text { A = repetitive } \\
\text { transcranial magnetic } \\
\text { stimulation on the left } \\
\text { primary motor cortex: } \\
10 \text { trains } 20 \mathrm{~Hz} \text { rTMS } \\
\text { using a figure-of-eight } \\
\text { coil for } 10 \text { min. } \\
\text { - Session } 2 \text { (Control) B = } \\
\text { median nerve } \\
\text { stimulation at wrist for } \\
10 \text { min. } \\
\text { - Session } 3 \text { (treatment A } \\
\text { repeated). }\end{array}$ & $\begin{array}{l}\text { Descriptive } \\
\text { (com- } \\
\text { pared\% } \\
\text { changes } \\
\text { across } \\
\text { A and B } \\
\text { condi- } \\
\text { tions } \\
\text { using } \\
\text { repeated- } \\
\text { measures } \\
\text { 1-way } \\
\text { ANOVA) }\end{array}$ & $\begin{array}{l}\text { Baseline, immediately } \\
\text { before and after each } \\
\text { session and then hourly } \\
\text { for } 8 \mathrm{~h}\end{array}$ & & $\begin{array}{l}\text { Immediately after the first } \\
\text { rTMS session, CRS-R } \\
\text { changed from } 13 \text { to } 19 \text {. } \\
\text { Improvement was } \\
\text { confirmed by CRS-R to } \\
\text { last for } 6 \mathrm{~h} \text {, and then } \\
\text { CRS-R score decreased } \\
\text { back to } 14 \text { in the seventh } \\
\text { hour of assessment. } \\
\text { On second administration } \\
\text { of treatment (A), CRS-R } \\
\text { score again increased, } \\
\text { this time from } 14 \text { to } 19 \text {, } \\
\text { again for } 6 \mathrm{~h} \text { when } \\
\text { CRS-R dropped to } 11 . \\
\text { At } 6 \text { month follow-up MCS } \\
\text { characteristics were the } \\
\text { same as pre-treatment. }\end{array}$ \\
\hline
\end{tabular}


TABLE 1

Continued.

\begin{tabular}{|c|c|c|c|c|c|c|}
\hline $\begin{array}{l}\text { Study (author, } \\
\text { date, country } \\
\text { of lead author) }\end{array}$ & $\begin{array}{l}\text { No. of participants/ } \\
\text { diagnosis/injury severity } \\
\text { on admission to study } \\
\text { (ranges, means/VS/ } \\
\text { MCS/RLAS/GCS)/ Time } \\
\text { post-injury/ Sex /Age } \\
\text { (range, mean) }\end{array}$ & $\begin{array}{l}\text { Treatment (including } \\
\text { duration and intensity of } \\
\text { treatment) }\end{array}$ & $\begin{array}{l}\text { Statistical } \\
\text { method to } \\
\text { determine } \\
\text { change on } \\
\text { CRS-R }\end{array}$ & $\begin{array}{l}\text { Assessment time points for } \\
\text { CRS-R }\end{array}$ & $\begin{array}{l}\text { Other outcome } \\
\text { measures at } \\
\text { concurrent } \\
\text { time-points }\end{array}$ & Findings \\
\hline $\begin{array}{l}\text { Sara, Pistoia, } \\
\text { Mura, } \\
\text { Onorati, \& } \\
\text { Govoni, } \\
2009 \\
\text { Italy }\end{array}$ & $\begin{array}{l}N=5 / \mathrm{ABI} \\
(\mathrm{SAH}=1, \mathrm{ICH}=1, \mathrm{TBI}= \\
2, \\
\text { post-anoxic } \\
\quad \text { encephalopathy }=1 \text { ) } \\
\text { VS } \\
\text { TPI: range }=6-12 \text { months, } \\
\quad M=9.2 \text { months, } \mathrm{SD}= \\
2.6 \text { months } \\
\text { Sex: } M=4, F=1 \\
\text { Age: range }=23-54 \\
\quad \text { years, } M=35.2 \text { years }\end{array}$ & $\begin{array}{l}\text { Intrathecal baclofen } \\
\text { therapy - } 100 \mu \mathrm{g} / \text { day } \\
\text { in continuous infusion. } \\
\text { The daily dose } \\
\text { progressively increased } \\
\text { over } 30 \text { days, with a } \\
\text { tapered infused dose } \\
\text { provided depending on } \\
\text { patient response. }\end{array}$ & $\begin{array}{l}\text { Visual } \\
\text { inspec- } \\
\text { tions of } \\
\text { graphs } \\
\text { and de- } \\
\text { scription } \\
\text { of raw } \\
\text { scores }\end{array}$ & $\begin{array}{l}\text { Baseline and } 6 \text { months } \\
\text { follow-up, (weekly } \\
\text { during treatment phase } \\
\text { - graph indicates } 40 \\
\text { weeks) }\end{array}$ & DRS & $\begin{array}{l}\text { Baseline CRS-R scores } \\
\text { ranged from } 5 \text { to } 7 \text {, } \\
\text { baseline DRS scores } \\
\text { ranged from } 22 \text { to } 24 \\
\text { (confirming diagnosis of } \\
\text { VS). } \\
\text { Clinical improvement in all } \\
\text { patients after } 2 \text { weeks of } \\
\text { active treatment. } \\
\text { All patients showed an } \\
\text { increase in CRS-R scores } \\
\text { with treatment. Over the } \\
\text { follow-up period, } 1 \\
\text { participant returned to } \\
\text { the starting state and the } \\
\text { other } 4 \text { participants } \\
\text { showed variable levels } \\
\text { of improvement. } \\
\text { Data: } \\
\text { Patient } 1: \text { CRS-R moved } \\
\text { from } 7 \text { to } 23 \text { then down } \\
\text { to } 9 \\
\text { Patient } 2: \text { CRS-R moved } \\
\text { from } 7 \text { to } 23 \\
\text { Patient } 3: \text { CRS-R moved } \\
\text { from } 6 \text { to } 21 \\
\text { Patient } 4: \text { CRS-R moved } \\
\text { from } 6 \text { to } 12 \text { Patient } 5 \text { : } \\
\text { CRS-R moved from } 5 \text { to } \\
11 .\end{array}$ \\
\hline
\end{tabular}


TABLE 1

Continued.

\begin{tabular}{|c|c|c|c|c|c|c|}
\hline $\begin{array}{l}\text { Study (author, } \\
\text { date, country } \\
\text { of lead author) }\end{array}$ & $\begin{array}{l}\text { No. of participants/ } \\
\text { diagnosis/iniury severity } \\
\text { on admission to study } \\
\text { (ranges, means/VS/ } \\
\text { MCS/RLAS/GCS)/ Time } \\
\text { post-injury/ Sex /Age } \\
\text { (range, mean) }\end{array}$ & $\begin{array}{l}\text { Treatment (including } \\
\text { duration and intensity of } \\
\text { treatment) }\end{array}$ & $\begin{array}{l}\text { Statistical } \\
\text { method to } \\
\text { determine } \\
\text { change on } \\
\text { CRS-R }\end{array}$ & $\begin{array}{l}\text { Assessment time points for } \\
\text { CRS-R }\end{array}$ & $\begin{array}{l}\text { Other outcome } \\
\text { measures at } \\
\text { concurrent } \\
\text { time-points }\end{array}$ & Findings \\
\hline $\begin{array}{l}\text { Schnakers } \\
\text { et al., } 2008 \\
\text { Belgium }\end{array}$ & $\begin{array}{l}N=1 / \text { Anoxic } \\
\text { MCS } \\
\text { TPI: Approx. } 2 \text { years } 7 \\
\text { months } \\
\text { Sex: } 0^{7} \\
\text { Age: } 23 \text { years }\end{array}$ & $\begin{array}{l}\text { Amantadine } 200 \mathrm{mg} / \text { day } \\
\text { during treatment phase } \\
\text { (ABAB design) } \\
\text { Baseline }(\mathrm{A} 1)=3 \text { weeks } \\
\text { Treatment time } 1(\mathrm{~B} 1)=6 \\
\text { weeks } \\
\text { Washout (A2) }=6 \text { weeks } \\
\text { Treatment time 2 }(\mathrm{B} 2)=6 \\
\text { weeks }\end{array}$ & $\begin{array}{l}\text { Descriptive } \\
\quad \text { (com- } \\
\text { parison } \\
\text { of mean } \\
\text { scores } \\
\text { during } \\
\text { baseline } \\
\text { and } \\
\text { treat- } \\
\text { ment } \\
\text { phases) }\end{array}$ & Weekly over 21 weeks & & $\begin{array}{l}\text { CRS-R scores changed from } \\
\text { baseline (A1) measures } \\
\text { (mean }=9.0, \mathrm{SD}=0.6 \text { ) } \\
\text { to treatment time } 1(\mathrm{~B} 1) \\
\text { measures (mean }=12.5, \\
\mathrm{SD}=2.4 \text { ). } \\
\text { Increases in the CRS scores } \\
\text { were maintained during } \\
\text { the washout (A2) and } \\
\text { treatment time 2 (B2) } \\
\text { phases. }\end{array}$ \\
\hline $\begin{array}{l}\text { Vargus- } \\
\text { Adams, } \\
\text { McMahon, } \\
\text { Michaud, } \\
\text { Bean, \& } \\
\text { Vinks, } \\
\text { 2010) USA }\end{array}$ & $\begin{array}{l}N=7 / \mathrm{ABI} \\
\text { (TBI }=5, C V A=1 \text {, anoxia } \\
=1 \text { ) } \\
\text { Initial } G C S \text { ranging from } 3 \\
\text { to } 6, M=4 \\
\text { TPI: } 4-10 \text { weeks } \\
\text { post-injury (median }=7 \\
\text { weeks) } \\
\text { Sex: } 0^{3}=6,9=1 \\
\text { Age: } 5-18 \text { years ( } M= \\
\text { 12.7) }\end{array}$ & $\begin{array}{l}\text { Each participant was } \\
\text { randomised to receive } \\
\text { treatment ( } 3 \text { weeks of } \\
\text { amantadine) first or } \\
\text { placebo ( } 3 \text { weeks } \\
\text { placebo) first, followed } \\
\text { by } 1 \text { week washout and } \\
\text { alternate condition ( } 3 \\
\text { weeks placebo or } 3 \\
\text { weeks amantadine) } \\
\text { followed by washout. } \\
\text { Treatment ( } 3 \text { weeks } \\
\text { amantadine): } \\
\text { Week } 1=\text { amantadine } 4 \\
\text { mg/kg/day divided into } \\
2 \text { equal doses }\end{array}$ & $\begin{array}{l}\text { Pearson } \\
\quad \text { correla- } \\
\text { tion }\end{array}$ & $\begin{array}{l}3 \text { times weekly to assess } \\
\text { clinical response }\end{array}$ & C/NCS & $\begin{array}{l}\text { No significant association } \\
\text { between average drug } \\
\text { concentrations and } \\
\text { slopes of the CRS-R ( } r= \\
0.424) \text { or CNCS ( } r= \\
-0.316) \text {. } \\
\text { The } 2 \text { participants with the } \\
\text { highest concentration of } \\
\text { amantadine (measured } \\
\text { via blood sampling) } \\
\text { demonstrated the 'most } \\
\text { favourable' recovery } \\
\text { slopes on amantadine, } \\
\text { as measured by the } \\
\text { CRS-R. }\end{array}$ \\
\hline
\end{tabular}


TABLE 1

Continued.

\begin{tabular}{|c|c|c|c|c|c|c|}
\hline $\begin{array}{l}\text { Study (author, } \\
\text { date, country } \\
\text { of lead author) }\end{array}$ & $\begin{array}{l}\text { No. of participants/ } \\
\text { diagnosis/injury severity } \\
\text { on admission to study } \\
\text { (ranges, means/VS/ } \\
\text { MCS/RLAS/GCS)/ Time } \\
\text { post-injury/ Sex /Age } \\
\text { (range, mean) }\end{array}$ & $\begin{array}{l}\text { Treatment (including } \\
\text { duration and intensity of } \\
\text { treatment) }\end{array}$ & $\begin{array}{l}\text { Statistical } \\
\text { method to } \\
\text { determine } \\
\text { change on } \\
\text { CRS-R }\end{array}$ & $\begin{array}{l}\text { Assessment time points for } \\
\text { CRS-R }\end{array}$ & $\begin{array}{l}\text { Other outcome } \\
\text { measures at } \\
\text { concurrent } \\
\text { time-points }\end{array}$ & Findings \\
\hline & & $\begin{array}{l}\text { Weeks } 2 \& 3= \\
\text { amantadine } 6 \\
\mathrm{mg} / \mathrm{kg} / \text { day divided into } \\
2 \text { equal doses } \\
\text { Placebo: } 3 \text {-week period } \\
\text { when placebo } \\
\text { administered }\end{array}$ & & & & \\
\hline $\begin{array}{l}\text { Whyte \& } \\
\text { Myers, } \\
2009 \\
\text { USA }\end{array}$ & $\begin{array}{l}N=15 / \mathrm{ABI}(\mathrm{TBI}=8, \\
\text { anoxia }=5, C V A=1, \\
\text { toxic encephalopathy }= \\
1) \\
M C S=3, \mathrm{VS}=12 \\
\text { TPI: } 1-278 \text { months, } \\
\text { median }=30.5 \text { momths } \\
\text { Sex: } \sigma^{7}=10, q=5 \\
\text { Age: } 22-59 \text { years } \\
(M=38 \text { years })\end{array}$ & $\begin{array}{l}\text { Zolpidem (hypnotic } \\
\text { medication) } 10 \mathrm{mg} \text { and } \\
\text { placebo treatment; } \\
1 \text { day zolpidem provided, } \\
1 \text { day placebo provided } \\
\text { (randomised cross-over } \\
\text { design) }\end{array}$ & $\begin{array}{l}\text { Visual } \\
\text { inspection, } \\
\text { repeated } \\
\text { measures } \\
\text { ANCOVA, } \\
\text { Wilcoxon } \\
\text { signed- } \\
\text { rank test } \\
\text { on change } \\
\text { of CRS } \\
\text { scores } \\
\text { between } 1 \\
\text { and } 2 \\
\text { hours post- } \\
\text { treatment }\end{array}$ & $\begin{array}{l}\text { Baseline and repeated } \\
\text { hourly for } 6 \text { hours on } \\
\text { each day (zolpidem day } \\
\text { and placebo day) }\end{array}$ & & $\begin{array}{l}1 \text { out of } 15 \text { had a } \\
\text { significant change in } \\
\text { CRS scores (via visual } \\
\text { inspection) in response } \\
\text { to drug administration. } \\
\text { In the other } 14 \text { participants } \\
\text { there was no significant } \\
\text { effect of the drug } \\
\text { compared to the } \\
\text { placebo. } \\
\text { In the other } 14 \\
\text { participants, changes } \\
\text { between baseline and } 1 \\
\text { hour, and baseline and } \\
2 \text { hours after drug } \\
\text { administration were not } \\
\text { significant. }\end{array}$ \\
\hline
\end{tabular}

Abbreviations for Tables 1-4: ABI, acquired brain injury; AVM, arterio-venous malformation; Ax, assessment; BIRU, Brain Injury Rehabilitation Unit; C/NCS, Coma Near Coma Scale; CRS, Coma Recovery Scale; CRS-R, Coma Recovery Scale-Revised; CVA, cerebro-vascular accident; DOCS, Disorders of Consciousness Scale; DRS, Disability Rating Scale; EEG,

electroencephalogram; GCS, Glasgow Coma Scale; GOS, Glasgow Outcome Scale; ICH, intracranial haemorrhage; ICU, intensive care unit; LOS, length of stay; M, mean; MCS, minimally conscious state; NS, not significant; NTBI, non-traumatic brain injury; OBI, other brain injury; PALOCS, Post Acute Levels of Consciousness Scale; PTA, post traumatic amnesia; RLAS, Ranchos Los Amigos Cognitive Levels of Functioning Scale; SAH, subarachnoid haemorrhage; SD, standard deviation; SMART, Sensory Modality Assessment and Rehabilitation Technique; SSAM, Sensory Stimulation Assessment Measure; TBI, traumatic brain injury; TPI, time post-injury; UK, United Kingdom; USA, United States of America; VS, vegetative state; WHIM, Wessex Head Injury Matrix; WNSSP, Western Neuro Sensory Stimulation Profile; $\sigma^{*}$, male; ; , female. 
TABLE 2

Summary of studies that have measured change using WNSSP and/or SMART

\begin{tabular}{|c|c|c|c|c|c|c|}
\hline $\begin{array}{l}\text { Study (author, } \\
\text { date, country } \\
\text { of lead author) }\end{array}$ & $\begin{array}{l}\text { No. of participants/ } \\
\text { diagnosis/ injury } \\
\text { severity on admission to } \\
\text { study (ranges, means/ } \\
\text { VS/MCS/RLAS/GCS)/ } \\
\text { Time post-injury/ Sex } \\
\text { /Age (range, mean) }\end{array}$ & $\begin{array}{l}\text { Treatment description } \\
\text { (duration and intensity) }\end{array}$ & $\begin{array}{l}\text { Statistical method to } \\
\text { determine change for } \\
\text { WNSSP and/or SMART }\end{array}$ & Assessment time-points & $\begin{array}{l}\text { Other outcome } \\
\text { measure/s at } \\
\text { concurrent time-points }\end{array}$ & Findings \\
\hline $\begin{array}{l}\text { Eilander et al., } \\
2009 \\
\text { Netherlands }\end{array}$ & $\begin{array}{l}N=3 \text { (part of a larger } \\
\text { study of } N=44, \\
\text { data are presented } \\
\text { for change following } \\
\text { treatment for } N=3 \text { ) } \\
\text { P1: TBI; TPI, } 39 \text { days at } \\
\text { initial; male; } 17 \\
\text { years old } \\
\text { P2: TBI; male; } 25 \text { years } \\
\text { old } \\
\text { P3: cerebral hypoxia; } \\
\text { TPI, } 56 \text { days at } \\
\text { initial; male; } 6 \text { years } \\
\text { old }\end{array}$ & $\begin{array}{l}\text { Early intensive } \\
\text { neuro-rehabilitation } \\
\text { programme }\end{array}$ & $\begin{array}{l}\text { Comparison of } \\
\text { admission and } \\
\text { discharge scores. } \\
\text { Graph of WNSSP raw } \\
\text { scores over repeated } \\
\text { measures for visual } \\
\text { analysis }\end{array}$ & $\begin{array}{l}\text { Twice weekly Ax from } \\
\text { admission to } \\
\text { discharge on each } \\
\text { measure }\end{array}$ & $\begin{array}{l}\text { PALOCS (categorises } \\
\text { patients from level } 1 \\
\text { coma to } 8 \\
\text { consciousness) }\end{array}$ & $\begin{array}{l}\text { P1: PALOCS 2-7 (195 } \\
\text { days post-admission) } \\
\text { P2: PALOCS 3-2 (1111 } \\
\text { days post-admission) } \\
\text { P3: PALOCS 3-6 (83 } \\
\text { days post-admission) } \\
\text { Concurrent } \\
\text { improvements shown } \\
\text { on WNSSP } \\
\text { graphically (unable to } \\
\text { report WNSSP scores } \\
\text { from graphs) }\end{array}$ \\
\hline $\begin{array}{l}\text { Hall, } \\
\text { MacDonald, } \\
\& \text { Young, } \\
1992 \\
\text { Canada }\end{array}$ & $\begin{array}{l}N=6 \\
\text { TBI } \\
\text { All GCS } 8 \text { or less (at } \\
\text { entry to study) } \\
\text { TPl: range } 11-23 \text { days } \\
\text { Sex: } 5 \sigma^{7} / 19 \\
\text { Age: range } 16-58 \\
\text { years }\end{array}$ & $\begin{array}{l}\text { Neurosurgical ICU. } \\
\text { Alternating weeks of } \\
\text { directed Specific } \\
\text { Directed Stimulation } \\
\text { (including } \\
\text { multisensory input) } \\
\text { and Non-Directed } \\
\text { Stimulation for half } \\
\text { hour daily for } 4 \\
\text { weeks } \\
\text { ABAB single-case } \\
\text { methodology }\end{array}$ & $\begin{array}{l}\text { Descriptive analysis of } \\
\text { mean WNSSP total } \\
\text { and subscale scores } \\
\text { for visual analysis }\end{array}$ & $\begin{array}{l}\text { Pre and post } 4 \\
\quad \text { treatment phases }\end{array}$ & $\begin{array}{l}\text { Rader subscale scores } \\
\text { RLAS level } \\
\text { GCS total }\end{array}$ & $\begin{array}{l}\text { Positive change in mean } \\
\text { WNSSP subscale } \\
\text { scores, total GCS, } \\
\text { Rader scale scores } \\
\text { and RLAS level } \\
\text { between pre- and } 4 \\
\text { weeks post-treatment. } \\
\text { Overall, the } 6 \text { subjects } \\
\text { on average improved } \\
\text { from } 20 \% \text { at the } \\
\text { beginning of } \\
\text { treatment to } 80 \% \text { at } \\
\text { end of treatment, } \\
\text { using total possible } \\
\text { scores on WNSSP. }\end{array}$ \\
\hline
\end{tabular}


TABLE 2

Continued.

\begin{tabular}{|c|c|c|c|c|c|c|}
\hline $\begin{array}{l}\text { Study (author, } \\
\text { date, country } \\
\text { of lead author) }\end{array}$ & $\begin{array}{l}\text { No. of participants/ } \\
\text { diagnosis/ injury } \\
\text { severity on admission to } \\
\text { study (ranges, means/ } \\
\text { VS/MCS/RLAS/GCS)/ } \\
\text { Time post-injury/ Sex } \\
\text { /Age (range, mean) }\end{array}$ & $\begin{array}{l}\text { Treatment description } \\
\text { (duration and intensity) }\end{array}$ & $\begin{array}{l}\text { Statistical method to } \\
\text { determine change for } \\
\text { WNSSP and/or SMART }\end{array}$ & Assessment time-points & $\begin{array}{l}\text { Other outcome } \\
\text { measure/s at } \\
\text { concurrent time-points }\end{array}$ & Findings \\
\hline $\begin{array}{l}\text { Lammi, Smith, } \\
\text { Tate, \& } \\
\text { Taylor, } \\
2005 \\
\text { Australia }\end{array}$ & $\begin{array}{l}N=18 \\
\text { TBI } \\
M C S \text { at initial, } \\
\text { Lowest } G C S M=4.29 \\
\quad(S D=1.49 ; \\
\text { median }=4.0) \\
\text { TPI: } 43.36 \text { months } \\
\text { (SD }=8.86 ; \text { median } \\
=43.88 \text { ) at } \\
\text { follow-up } \\
\text { Sex: } 14 \sigma^{7} / 4 \% \\
\text { Age at injury: } M= \\
37.89 \text { (SD }=14.76 ; \\
\text { median }=37 \text { ) }\end{array}$ & $\begin{array}{l}\text { All received specialist } \\
\text { inpatient brain injury } \\
\text { rehabilitation }\end{array}$ & $\begin{array}{l}\text { Wilcoxon signed-rank } \\
\text { test examined } \\
\text { changes over time for } \\
\text { WNSSP total and } \\
\text { subscale scores. } \\
\text { Bonferri adjustments } \\
\text { to prevent type I error } \\
\text { rate ( } p \text { set at less than } \\
0.005) \text {. }\end{array}$ & $\begin{array}{l}\text { WNSSP on admission } \\
\text { and follow-up 2-5 } \\
\text { years post-injury }\end{array}$ & & $\begin{array}{l}\text { Significant improvement } \\
\text { seen between initial } \\
\text { WNSSP }[M=35.56 ; \\
\text { median }=32.5 \\
\text { (SD }=22.43)] \text { vs } \\
2-5 \text {-year follow-up } \\
{[M=101.67 ;} \\
\text { median }=112 \text { (SD = } \\
23.88)] ; \\
p=0.000 \text {. All } \\
\text { subscales showed } \\
\text { significant } \\
\text { improvement, with } \\
\text { exception of auditory } \\
\text { response ( } p= \\
\text { O.006). } \\
\text { Cohen's } \mathbf{d}=\mathbf{2 . 8 2}\end{array}$ \\
\hline $\begin{array}{l}\text { Patrick et al., } \\
2009 \\
\text { USA }\end{array}$ & $\begin{array}{l}N=10 \\
\text { TBI } \\
\text { Initial GCS } M=3 \text { at } \\
\text { emergency and RLAS } \\
\text { I, II or III for }>30 \\
\text { days post-injury } \\
\text { TPI: } M=66 \text { days } \\
\text { Sex: } 6 \% / 4 \sigma^{7} \\
\text { Age: } M=16.7 \text { years } \\
\text { (range } 8-21 \text { years) }\end{array}$ & $\begin{array}{l}\text { Inpatient rehabilitation } \\
\text { unit. } \\
\text { 6-week medication } \\
\text { protocol through } \\
\text { dopaminergic } \\
\text { agents (pramipexole } \\
\text { or amantadine). } \\
\text { 8-week duration (2 } \\
\text { weeks prior to } \\
\text { medication, } 6 \text { weeks } \\
\text { on medication) }\end{array}$ & $\begin{array}{l}\text { WNSSP total score } \\
\text { baseline and last } \\
\text { scores were tabulated } \\
\text { for each participant. } \\
\text { Correlational analysis } \\
\text { using a linear mixed } \\
\text { model to explore } \\
\text { association between } \\
3 \text { participation } \\
\text { ratings and WNSSP. }\end{array}$ & $\begin{array}{l}\text { Within } 24 \mathrm{~h} \text { of } \\
\text { admission; } 24 \mathrm{~h} \\
\text { before medication } \\
\text { trial; once weekly } \\
\text { during medication } \\
\text { trial; during } \\
\text { withdrawal } \\
\text { medication. } \\
\text { For all but } 3 \\
\text { participants, the last } \\
\text { score was collected } \\
\text { at week } 8 \text { (week } 7 \\
\text { for } 2 \text { participants, } \\
\text { week } 5 \text { for } 1 \\
\text { participant).' }\end{array}$ & $\begin{array}{l}\text { Participation was also } \\
\text { rated (score given } \\
\text { for arousal, } \\
\text { communication and } \\
\text { awareness derived } \\
\text { from video analysis } \\
\text { of behaviours } \\
\text { observed during } \\
\text { therapy) }\end{array}$ & $\begin{array}{l}\text { 9/10 improved } \\
\text { (variable levels of } \\
\text { improvement). } \\
\text { WNSSP and } \\
\text { communication } \\
\text { ratings highly } \\
\text { correlated (as } \\
\text { WNSSP improved, } \\
\text { communication } \\
\text { observed during } \\
\text { therapeutic } \\
\text { participation } \\
\text { improved). }\end{array}$ \\
\hline
\end{tabular}


TABLE 2

Continued.

\begin{tabular}{|c|c|c|c|c|c|c|}
\hline $\begin{array}{l}\text { Study (author, } \\
\text { date, country } \\
\text { of lead author) }\end{array}$ & $\begin{array}{l}\text { No. of participants/ } \\
\text { diagnosis/ injury } \\
\text { severity on admission to } \\
\text { study (ranges, means/ } \\
\text { VS/MCS/RLAS/GCS)/ } \\
\text { Time post-injury/ Sex } \\
\text { /Age (range, mean) }\end{array}$ & $\begin{array}{l}\text { Treatment description } \\
\text { (duration and intensity) }\end{array}$ & $\begin{array}{l}\text { Statistical method to } \\
\text { determine change for } \\
\text { WNSSP and/or SMART }\end{array}$ & Assessment time-points & $\begin{array}{l}\text { Other outcome } \\
\text { measure/s at } \\
\text { concurrent time-points }\end{array}$ & Findings \\
\hline & & & & & & $\begin{array}{l}\text { M, SD and Cohen's } d \\
\text { calculated from } \\
\text { tabulated raw scores } \\
\text { and baseline and end } \\
\text { of study. } \\
\text { Baseline: } M=11.4, S D \\
=8.54, \text { range } 2-30 . \\
\text { End: } M=52.8, S D= \\
42.20 \text {, range }=7- \\
-113 \text {. } \\
\text { Cohen's } d=1.36\end{array}$ \\
\hline $\begin{array}{l}\text { Smith et al., } \\
2001 \\
\text { Australia }\end{array}$ & $\begin{array}{l}N=25 \text { (total sample } \\
\text { size) } \\
\text { TBI } \\
\text { Average lowest } \mathrm{GCS} \\
(n=18,4.5, \mathrm{SD}= \\
1.62 \text { ) } \\
\text { TPI on admission: } \mathrm{M}= \\
52.3 \text { days (range } \\
17-216 \text { days) } \\
\text { Initial Ax } M=9.3 \text { days } \\
\text { after admission } \\
\text { Sex }=20 \% / 5 \sigma^{7} \\
\text { Age at iniury: } M= \\
24.4 \text { (SD }=19.17 \text { ) }\end{array}$ & $\begin{array}{l}\text { Inpatient brain injury } \\
\text { rehabilitation unit }\end{array}$ & $\begin{array}{l}\text { Wilcoxon tests } \\
\text { comparing total and } \\
\text { subscale scores on } \\
\text { adjacent and first to } \\
\text { fourth test occasion. } \\
\text { Administration WNSSP } \\
\text { repeated at regular } \\
\text { intervals determined } \\
\text { clinically on } \\
\text { individual basis. }\end{array}$ & $\begin{array}{l}\text { Subset } 12 / 25 \\
\text { assessed using } \\
\text { WNSSP on } 4 \text { or } \\
\text { more occasions } \\
\text { examined in detail }\end{array}$ & WNSSP & $\begin{array}{c}\text { Time 1: } M=28.25 \\
\text { SD }=26.66 \\
\text { Time 2: } M=32.83 \\
\text { SD }=20.23 \\
\text { Time } 3: M-38.33 \\
S D=28.47 \\
\text { Time } 4: M=41.67 \\
S D=27.20 \\
\text { No significant } \\
\text { differences between } \\
\text { total scores on either } \\
\text { adjacent test } \\
\text { occasions or when } \\
\text { Time } 1 \text { and Time 4 } \\
\text { scores compared. } \\
\text { Cohen's } \boldsymbol{d}=\mathbf{0 . 4 9} \\
\text { (Time } \mathbf{1} \text { vs Time 4) }\end{array}$ \\
\hline
\end{tabular}


TABLE 2

Continued.

No. of participants/

diagnosis/ injury

severity on admission to

study (ranges, means/

Study (author, VS/MCS/RLAS/GCS)/

date, country Time post-injury/Sex

/Age (range, mean)

Statistical method to

determine change for

Other outcome

of lead author

(duration and intensity)

WNSSP and/or SMART

measure/s at

Wilson \& Gill- $\quad N=30$

Thwaites, $\quad(\mathrm{TB})=15$, hypoxia $=$

2000

5 , other $A B \mid=10$ )

Specialist BIRU for

patients in VS.

SMART Sensory

ported mean, SD and

WNSSP: 1 week after

Independent

admission, 2 months

and SMART scores

$(N=30), 4$ months

stimulation treatment for total group and

TPI: $M=14.3$ months (range 2.1-59.7)

Sex: $11 \% / 190^{\circ}$

the

$N=22), 6$ months

$(N=9)$

for duration of emerged/emerged

admission.

later and

SMART: Twice daily for

non-emerged group

(SD $=11.8$

16.4-57.8)

Sample divided into 3

groups based on

emergence from VS:

$7 / 30$ emerged from VS during study

('Emerged group')

$6 / 30$ emerged from VS

later (at time

analysis) ('Emerged

later group')

17/30 non-emerged

(remained VS at time

analysis)

at 4 time points.

Tested significance of

changes in total

WNSSP and SMART

scores over first 3

assessments for 3

groups

lemerged/emerged

later and

non-emerged) using

Friedman's ANOVA.

admission

assessment by team WNSSP

members of

emergence in

accordance with

Jennet and Plum's

definition of VS

Uennett \& Plum,

1972).

Time 1 $(N=30): M=$ $15.3(S D=8.3)$

Time $2(N=30): M=$ $22.7(S D=14.9)$

Time $3(N=22): M=$

$27.4(S D=19.4)$

Time $4(N=9): M=$

$30.1(S D=25.3)$

Cohen's $d=0.61$

(time 1-time 2)

Cohen's $d=\mathbf{0 . 8 6}$

(time 1-time 3) 
TABLE 2

Continued.

No. of participants/

diagnosis/ injury

severity on admission to

study (ranges, means/

Study (author, VS/MCS/RLAS/GCS)/

date, country Time post-injury/Sex

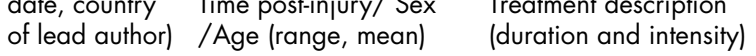

Statistical method to

Treatment description determine change for

WNSSP and/or SMART Assessment time-points

Other outcome

measure/s at

concurrent time-points

Findings

Cohen's $d=1.07$

(time 1-ime 4)

Whole group data SMART

Time $1(N=30): M=$ $16.0(\mathrm{SD}=3.5)$

Time $2(N=30): M=$

$17.2(\mathrm{SD}=4.7)$

Time $3(N=22): M=$ $19.5(S D=6.1)$

Time $4(N=9): M=$

$21.4(\mathrm{SD}=6.6)$

Cohen's $d=0.29$

(time 1-time 2)

Cohen's $d=0.73$

(time 1-time 3)

Cohen's $d=1.24$

(time 1-time 4)

Friedman's ANOVA

Results

Emerged group:

WNSSP: $X=7.0, \mathrm{df}=$ $2, p=0.030$.

SMART X $=13.0, \mathrm{df}=$

$2, p=0.002$

Emerged later group

WNSSP X $=8.0, \mathrm{df}=$

$2, p=0.018$

SMART $X=6.86, \mathrm{df}=$

$2, p=0.032$

NS for non-emerged group 
TABLE 3

Summary of studies that have measured change using the WHIM

\begin{tabular}{|c|c|c|c|c|c|c|}
\hline $\begin{array}{l}\text { Study (author, } \\
\text { date, country } \\
\text { of lead author) }\end{array}$ & $\begin{array}{l}\text { No. of participants/ } \\
\text { diagnosis/ injury } \\
\text { severity on admission to } \\
\text { study (ranges, means/ } \\
\text { VS/MCS/RLAS/GCS)/ } \\
\text { Time post-injury/ Sex } \\
\text { /Age (range, mean) }\end{array}$ & $\begin{array}{l}\text { Treatment description } \\
\text { (duration and intensity) }\end{array}$ & $\begin{array}{l}\text { Statistical method to } \\
\text { determine change on } \\
\text { WHIM }\end{array}$ & $\begin{array}{l}\text { Assessment time-points } \\
\text { for WHIM }\end{array}$ & $\begin{array}{l}\text { Other } \\
\text { outcome } \\
\text { measures at } \\
\text { concurrent } \\
\text { time-points }\end{array}$ & Findings \\
\hline $\begin{array}{l}\text { Crossley et al., } \\
2005 \\
\text { UK }\end{array}$ & $\begin{array}{l}N=1 \mathrm{TBI}+\text { Age: } 80 \\
\text { years }\end{array}$ & $\begin{array}{l}\text { Followed up from ICU } \\
\text { through to inpatient } \\
\text { rehabilitation } \\
\text { (admitted inpatient } \\
\text { rehabilitation at } 10 \\
\text { weeks post-injury for } \\
3 \text { month LOS). }\end{array}$ & $\begin{array}{l}\text { Summary using } \\
\text { scatterplot of WHIM } \\
\text { total behaviours and } \\
\text { highest rank } \\
\text { behaviour scores over } \\
\text { time }\end{array}$ & $\begin{array}{l}2 \text { weeks post-admission } \\
\text { to ICU, daily } \\
\text { monitoring for } 16 \\
\text { weeks, then } 2-3 \text { times } \\
\text { weekly until discharge } \\
\text { home (approximately } \\
5.5 \text { months } \\
\text { post-injury) }\end{array}$ & & $\begin{array}{l}\text { Visual inspection } \\
\text { indicates rapid } \\
\text { recovery from coma } \\
\text { according to WHIM } \\
\text { at } 4 \text { weeks, gradual } \\
\text { recovery with } \\
\text { increasing alertness } \\
\text { and lucidity over } \\
\text { following } 8 \text { weeks } \\
\text { until week } 12 \text { where } \\
\text { there was consistent } \\
\text { orientation and intact } \\
\text { language functions } \\
\text { with plateau at week } \\
14 \text { (emerged from } \\
\text { PTA) }\end{array}$ \\
\hline $\begin{array}{l}\text { Elliot et al., } \\
2005 \\
\text { UK }\end{array}$ & $\begin{array}{l}N=12 \text { VS: } N=5, \text { and } \\
M C S: N=7 \text { Sex: } \\
8 \sigma^{\circ} / 4 q \text { Age: } M= \\
\text { 49, range 19-71 }\end{array}$ & $\begin{array}{l}\text { Lying versus standing } \\
\text { ( } 20 \text { minutes) in tilt } \\
\text { table (postural } \\
\text { change) }\end{array}$ & $\begin{array}{l}\text { Comparison } \\
\text { (non-parametric) of } \\
\text { median highest } \\
\text { ranked behaviour } \\
\text { and median total } \\
\text { number of behaviours } \\
\text { for lying and standing } \\
\text { conditions. }\end{array}$ & $\begin{array}{l}\text { Repeated observations } \\
\text { over a l-week period. }\end{array}$ & & $\begin{array}{l}8 / 12 \text { consistently } \\
\text { improved } \\
\text { highest-ranked } \\
\text { behaviour ( } p= \\
0.008) \text { and total } \\
\text { number of behaviours } \\
(p=0.013) \text { in } \\
\text { standing when } \\
\text { compared to sitting. } \\
3 / 12-\text { no change. } \\
1 / 12 \text { improved only } \\
\text { in highest-ranked } \\
\text { behaviour observed. }\end{array}$ \\
\hline
\end{tabular}


TABLE 3

Continued.

\begin{tabular}{|c|c|c|c|c|c|c|}
\hline $\begin{array}{l}\text { Study (author, } \\
\text { date, country } \\
\text { of lead author) }\end{array}$ & $\begin{array}{l}\text { No. of participants/ } \\
\text { diagnosis/ injury } \\
\text { severity on admission to } \\
\text { study (ranges, means/ } \\
\text { VS/MCS/RLAS/GCS)/ } \\
\text { Time post-injury/ Sex } \\
\text { /Age (range, mean) }\end{array}$ & $\begin{array}{l}\text { Treatment description } \\
\text { (duration and intensity) }\end{array}$ & $\begin{array}{l}\text { Statistical method to } \\
\text { determine change on } \\
\text { WHIM }\end{array}$ & $\begin{array}{l}\text { Assessment time-points } \\
\text { for WHIM }\end{array}$ & $\begin{array}{l}\text { Other } \\
\text { outcome } \\
\text { measures at } \\
\text { concurrent } \\
\text { time-points }\end{array}$ & Findings \\
\hline $\begin{array}{l}\text { Wilson, } \\
\text { Coleman \& } \\
\text { Pickard, } \\
2008 \\
\text { UK }\end{array}$ & $\begin{array}{l}N=21 \mathrm{VS}(N=10) \\
M C S(N=11)\end{array}$ & $\begin{array}{l}\text { Effect of lying versus } \\
\text { standing (tilt table) } \\
\text { compared }\end{array}$ & $\begin{array}{l}\text { Wilcoxon matched-pairs } \\
\text { signed ranks test. }\end{array}$ & $\begin{array}{l}\text { Ax lying in bed, } \\
\text { standing, then lying in } \\
\text { bed }\end{array}$ & & $\begin{array}{l}\text { Higher-ranked } \\
\text { behaviour in standing } \\
\text { compared to sitting ( } p \\
<0.008) \text {. Using } \\
\text { tabulated raw data } \\
\text { reported for } 12 \\
\text { participants, the } \\
\text { following were } \\
\text { calculated: Standing: } \\
M=17.33, S D= \\
13.73, \text { range } 1-43 \\
\text { Supine: } M=28.5 \\
S D=13.26 \text {, range } \\
4-49 \text { Cohen's } \mathbf{d}= \\
\mathbf{0 . 8 3}\end{array}$ \\
\hline $\begin{array}{l}\text { Wilson, Elder, } \\
\text { McCradden } \\
\text { \& Caldwell, } \\
2009 \\
\text { UK }\end{array}$ & $\begin{array}{c}N=20 \text { (TBI: } N=14 ; \\
\text { hypoxic brain injury: } \\
N=7 ; \text {; } S A H: N=2 \text { ) } \\
\text { Either in } \mathrm{VS} \text { or } M C S \\
\text { on admission TPI on } \\
\text { admission: } M=146 \\
\text { days, } S D=78.5 \\
\text { days, range } 47-379 \\
\text { Sex: } 130^{\circ} / 7 \text { \% Age: } \\
M=38, S D=14.5, \\
\text { range } 15-61 \text { years }\end{array}$ & $\begin{array}{l}\text { Systematic review of } \\
\text { records of patients } \\
\text { who were assessed } \\
\text { during their admission } \\
\text { to Forster Green } \\
\text { Hospital and then to } \\
\text { the regional acquired } \\
\text { brain injury unit }\end{array}$ & $\begin{array}{l}\text { Wilcoxon signed rank } \\
\text { analysis of WHIM } \\
\text { total number } \\
\text { behaviours and } \\
\text { WHIM highest rank } \\
\text { behaviour comparing } \\
\text { first and final WHIM } \\
\text { assessment scores ( } n \\
=2 \text { excluded from } \\
\text { analysis) }\end{array}$ & $\begin{array}{l}\text { Initial }(M=156 \text { days } \\
\text { post-insult, } S D= \\
76.4) \text { and final } \\
\text { assessment scores } \\
\text { (assessed on average } \\
5 \text { times during } \\
\text { admission, range } \\
1-15) \text { over LOS (M }= \\
186 \text { days, } S D= \\
155.8, \text { range } \\
15-612 \text { days) }\end{array}$ & & $\begin{array}{l}\text { Significant } \\
\text { improvements in total } \\
\text { number of behaviours } \\
\text { recorded ( } z= \\
-3.62, p<0.01) \\
\text { and highest-ranked } \\
\text { behaviour }(z= \\
-3.46, p=0.001) \text {. } \\
\text { Tabulated initial and } \\
\text { final scores on WHIM } \\
\text { highest-ranked } \\
\text { behaviours used to } \\
\text { calculate: Initial: } M= \\
21.05, S D=11.78,\end{array}$ \\
\hline
\end{tabular}


TABLE 3

Continued.

\begin{tabular}{|c|c|c|c|c|c|c|}
\hline $\begin{array}{l}\text { Study (author, } \\
\text { date, country } \\
\text { of lead author) }\end{array}$ & $\begin{array}{l}\text { No. of participants/ } \\
\text { diagnosis/ injury } \\
\text { severity on admission to } \\
\text { study (ranges, means/ } \\
\text { VS/MCS/RLAS/GCS)/ } \\
\text { Time post-injury/ Sex } \\
\text { /Age (range, mean) }\end{array}$ & $\begin{array}{l}\text { Treatment description } \\
\text { (duration and intensity) }\end{array}$ & $\begin{array}{l}\text { Statistical method to } \\
\text { determine change on } \\
\text { WHIM }\end{array}$ & $\begin{array}{l}\text { Assessment time-points } \\
\text { for WHIM }\end{array}$ & $\begin{array}{l}\text { Other } \\
\text { outcome } \\
\text { measures at } \\
\text { concurrent } \\
\text { time-points }\end{array}$ & Findings \\
\hline & & & & & & $\begin{array}{l}\text { range } 6-52 \text { Final: } \\
M=39.27, S D= \\
13.49, \text { range } 17-58 \\
\text { Cohen's } \boldsymbol{d}=\mathbf{1 . 4 4}\end{array}$ \\
\hline $\begin{array}{l}\text { Wilson, } \\
\text { Harpur, } \\
\text { Watson, \& } \\
\text { Morrow, } \\
2003 \\
\text { UK }\end{array}$ & $\begin{array}{l}N=7 \text { (study included } \\
N=13, N=7 \\
\text { underwent serial } \\
\text { assessment) } \\
\text { Cerebral hypoxia } \\
\text { TPI at follow-up: } M= \\
21.8 ; \text { range } 15-32 \\
\text { months } \\
\text { Sex: } 10 \sigma^{7} / 3 \circ \\
\text { Age at injury: } M= \\
43.2, S D=14.27, \\
\text { range } 25-58 \text { years } \\
\text { Age at assessment: } M= \\
44.28, \\
\text { SD }=14.13 \text {, range } \\
26-59 \text { years }\end{array}$ & $\begin{array}{l}\text { All patients referred to } \\
\text { inpatient or } \\
\text { community brain } \\
\text { injury service for } \\
\text { assessment, } \\
\text { rehabilitation and/or } \\
\text { management advice }\end{array}$ & $\begin{array}{l}\text { Descriptive analysis } \\
\text { (tabulated scores for } \\
\text { each case) }\end{array}$ & $\begin{array}{l}\text { Serial assessment over } \\
\text { period of at least } 2 \\
\text { years (range: } 3-27 \\
\text { months) }\end{array}$ & $\begin{array}{l}\text { RLAS } \\
\text { GCS }\end{array}$ & $\begin{array}{l}\text { GCS (4 same; } 3 \\
\text { improved); RLAS (4 } \\
\text { same; } 3 \text { improved } \\
\text { concurrently with } \\
\text { GCS); all improved } \\
\text { rank number and } \\
\text { highest behaviour on } \\
\text { WHIM. Calculated } \\
\text { means and SD for } \\
\text { initial and current } \\
\text { WHIM scores from } \\
\text { raw scores in paper. } \\
\text { WHIM highest-ranked } \\
\text { behaviour: Initial: } \\
\text { M }=16.85, \text { SD }= \\
16.38 \text { End: } M= \\
27.71, \text { SD = } 21.21 \\
\text { Cohen's } \boldsymbol{d}=\mathbf{0 . 5 7} \\
\text { WHIM total number } \\
\text { behaviours: Initial: } \\
\text { M=22.71, SD = } \\
22.9 \text { End: } M= \\
33.71 \text {, } \\
\text { SD = 21.8 Cohen's } \\
\text { d=0.49 }\end{array}$ \\
\hline
\end{tabular}

See the footnote to Table 1 for abbreviations. 
and RLAS with improvement on the WHIM. The WHIM indicated improvements in all seven cases whereas the GCS and RLAS indicated concurrent improvements in only four of the seven cases (Wilson et al., 2003).

Very few articles measured change on the SSAM $(n=1)$ or DOCS $(n=2)$ (Table 4$)$. The two papers relating to the DOCS descriptively reported change during inpatient brain injury rehabilitation of a large sample showing change over time (Pape et al., 2009) and three detailed case studies showing change over time and responsiveness to medication changes influencing arousal (Pape, Senno, Guernon, \& Kelly, 2005b). The regular serial assessment over time using the DOCS in the three cases highlights the value of regular assessment for intervention planning to maximise consciousness (Pape et al., 2005b). The study by Davis and Gimenez (2003) used statistical analysis to demonstrate greater change in an intervention group $(N=9)$ receiving auditory sensory stimulation compared to a small control group $(N=3)$, and showed greater sensitivity than the GCS and RLAS in demonstrating these changes.

\section{Discussion}

This review has summarised the 24 studies identified in our literature search which have used the CRS-R, DOCS, SMART, SSAM, WHIM and WNSSP to measure change over time in response to treatment. The identified studies mostly aimed to investigate the efficacy of treatment programmes over time, or describe/predict outcomes by conducting follow-up assessments at varying lengths of time after initial assessment and injury. Overall, no study that was identified aimed specifically to examine the responsiveness of the measures. The quality of the data on responsiveness is limited across all measures because the majority of studies reported primarily descriptive analysis and only 8 of the 25 studies reported statistical analysis of change. However, the identified studies did provide some evidence for the ability of these measures to detect change following treatment. The CRS-R, WNSSP and WHIM have established responsiveness to change, based on the findings of more than one study that have incorporated statistical analysis in order to demonstrate significant change. Additionally, all of these measures had at least one study where it was possible to calculate the effect size of the change using Cohen's $d$. Effect sizes for all measures were large. The SMART has demonstrated responsiveness to change based on a single study using statistical analysis to detect change and a large effect size over the course of the entire study. The SSAM has moderate levels of evidence for responsiveness from the findings of one study incorporating statistical analysis of change; however, it was not possible to calculate effect size. The DOCS has preliminary evidence for responsiveness in the form of a study that did not statistically analyse change in scores over measurement time-points but did, however, use descriptive analysis to demonstrate changes in data over time.

Despite the need to establish the responsiveness of available behavioural assessments of people with disordered consciousness, there are obvious limitations associated with relying on observational assessment of consciousness in isolation. For example, the constellation of impairments resulting from the related injury may mean that the person is unable to demonstrate their underlying ability or awareness on some assessment items. For example, physical limitations preventing movement or speech may prevent demonstration of a response. Particularly in these cases, as discussed in a review of the behavioural evaluation of consciousness by Majerus et al. (2005), individualised assessment approaches such as those outlined by Whyte and colleagues $(1995,1999)$ are necessary to detect subtle but clinically significant responses. Responsiveness can be assessed individually if a voluntary response is present. This can be determined by comparing the frequency of the behaviour between baseline and other phases, where the person is required to produce the behaviour (i.e., in response to a command or condition) (Majerus, Gill-Thwaites, Andrews, \& Laureys, 2005). An example of this is a series of studies by Lancioni and colleagues $(2009,2011)$, where single-case experimental designs are used to compare the frequency of individual responses (i.e., eye blink to activate an optic sensor microswitch) between baseline and intervention phases. These studies, which used an individualised assessment approach, were able to provide evidence that, in these cases, there was a clinically meaningful change (i.e., the person was able to learn to use micro-switch technology to request assistance from a caregiver) (Lancioni et al., 2009, 2011). These studies also highlight the value of technology in enabling access and overcoming limitations, which may prevent a person from interacting with their environment and being able to give a response (Lancioni et al., 2009, 2011). Also, it is important to consider the use of other assessment techniques, such as functional magnetic resonance imaging (Rodriguez Moreno, Schiff, Giacino, Kalmar, \& Hirsch, 2010) and functional electroencephalography (Cruse et al., 2012), to ensure a comprehensive approach to assessing consciousness. 
TABLE 4

Summary of studies that have measured change using SSAM or DOCS

\begin{tabular}{|c|c|c|c|c|c|c|}
\hline $\begin{array}{l}\text { Study (author, } \\
\text { date, country } \\
\text { of lead author) }\end{array}$ & $\begin{array}{l}\text { No. of participants/ } \\
\text { diagnosis/ injury } \\
\text { severity on admission to } \\
\text { study (ranges, means/ } \\
\text { VS/MCS/RLAS/GCS)/ } \\
\text { Time post-injury/ Sex } \\
\text { /Age (range, mean) }\end{array}$ & $\begin{array}{l}\text { Treatment description } \\
\text { (duration and intensity) }\end{array}$ & $\begin{array}{l}\text { Statistical method to } \\
\text { determine change on } \\
\text { SSAM or DOCS }\end{array}$ & Assessment time-points & $\begin{array}{l}\text { Other } \\
\text { outcome } \\
\text { measures at } \\
\text { concurrent } \\
\text { time-points }\end{array}$ & Findings \\
\hline $\begin{array}{l}\text { Davis \& } \\
\text { Gimenez, } \\
2003 \\
\text { USA }\end{array}$ & $\begin{array}{l}N=12 \text { (9 intervention; } \\
3 \text { controls) } \\
\text { TBI } \\
\text { RLAS level I, II or III } \\
\text { TPI: up to } 3 \text { days at } \\
\text { initial Ax } \\
\text { Sex: All } \sigma^{7} \\
\text { Age: } M=30 \text { (range } \\
17-55 \text { ) }\end{array}$ & $\begin{array}{l}\text { Control and intervention } \\
\text { groups } \\
\text { (non-randomised) } \\
\text { received routine } \\
\text { inpatient rehab. } \\
\text { Intervention group } \\
\text { also received } \\
\text { structured auditory } \\
\text { sensory stimulation } \\
\text { (5-8 sessions daily) } \\
\text { for up to } 7 \text { days; 5-8 } \\
\text { sessions daily for } \\
5-15 \text { minutes per } \\
\text { session }\end{array}$ & $\begin{array}{l}t \text {-test comparing mean } \\
\text { SSAM baseline and } \\
\text { end of treatment } \\
\text { scores }\end{array}$ & $\begin{array}{l}\text { Baseline and } \\
\text { post-treatment }\end{array}$ & $\begin{array}{l}\text { GCS } \\
\text { RLAS } \\
\text { DRS }\end{array}$ & $\begin{array}{l}\text { Mean change raw score } \\
\text { SSAM intervention, } \\
11 \text { versus control } 0.3 \\
(p=0.015) ; \text { GCS } \\
\text { intervention, } 3.3 \\
\text { versus control } 1.0 \\
(p=0.278) ; \text { RLAS } \\
\text { intervention, } 1.2 \\
\text { versus control no } \\
\text { change; DRS } \\
\text { intervention, } 3.7 \\
\text { versus } 0.3(p= \\
\text { 0.0005) }\end{array}$ \\
\hline $\begin{array}{l}\text { Pape et al., } \\
2005 b \\
\text { USA }\end{array}$ & $\begin{array}{l}N=4 \\
\text { All TBI } \\
\text { P1: initial GCS = } 3 ; \\
\quad \text { female; age } 20 \text { years } \\
\text { (TBI and hypoxic } \\
\text { injury) } \\
\text { P2: initial GCS = } 3 \text {; } \\
\text { male; age } 18 \text { years } \\
\text { P3: female; age } 72 \\
\text { years } \\
\text { P4: initial GCS = 5, } \\
\text { male, age } 33 \text { years } \\
\text { Admission to inpatient } \\
\text { rehab discharge } \\
\text { (days post-injury): } \\
\text { P1: } 42-157 \\
\text { P2: } 32-66 \\
\text { P3: } 27-100 \\
\text { P4: } 116-137\end{array}$ & $\begin{array}{l}\text { Multidisciplinary } \\
\text { inpatient } \\
\text { rehabilitation } \\
\text { (P1: } 3.5 \text { hours; P2: } 2.61 \\
\text { hours and P3: } 2.2 \\
\text { hours therapy per day } \\
\text { during inpatient } \\
\text { rehabilitation). Onset } \\
\text { and withdrawal of } \\
\text { various } \\
\text { pharmacological } \\
\text { interventions: } \\
\text { P1: hypertonicity } \\
\text { management } \\
\text { P2: methylphenidate } \\
\text { P3: epilepsy } \\
\text { management. }\end{array}$ & $\begin{array}{l}\text { Scatterplots and bar } \\
\text { graphs of DOCS } \\
\text { scores by days after } \\
\text { injury noting on } \\
\text { graph specific timings } \\
\text { for introduction/ } \\
\text { withdrawal of specific } \\
\text { interventions }\end{array}$ & $\begin{array}{l}\text { Weekly DOCS } \\
\text { evaluations for } 6 \\
\text { weeks following } \\
\text { admission followed } \\
\text { by regular } \\
\text { evaluations for } \\
\text { duration of inpatient } \\
\text { rehabilitation, then } \\
\text { monthly evaluations } \\
\text { for up to } 1 \text { year } \\
\text { post-discharge }\end{array}$ & & $\begin{array}{l}\text { Scatterplots indicated } \\
\text { improved DOCS } \\
\text { scores between end } \\
\text { and initial Ax, with } \\
\text { fluctuating levels of } \\
\text { consciousness in } \\
\text { between end and } \\
\text { initial Ax associated } \\
\text { with medication } \\
\text { events. Examples } \\
\text { included improved } \\
\text { DOCS scores with: } \\
\text { P1: change of } \\
\text { hypertonicity } \\
\text { medication } \\
\text { P2: withdrawal of } \\
\text { methylphenidate }\end{array}$ \\
\hline
\end{tabular}


TABLE 4

Continued.

\begin{tabular}{|c|c|c|c|c|c|c|}
\hline $\begin{array}{l}\text { Study (author, } \\
\text { date, country } \\
\text { of lead author) }\end{array}$ & $\begin{array}{l}\text { No. of participants/ } \\
\text { diagnosis/ injury } \\
\text { severity on admission to } \\
\text { study (ranges, means/ } \\
\text { VS/MCS/RLAS/GCS)/ } \\
\text { Time post-injury/ Sex } \\
\text { /Age (range, mean) }\end{array}$ & $\begin{array}{l}\text { Treatment description } \\
\text { (duration and intensity) }\end{array}$ & $\begin{array}{l}\text { Statistical method to } \\
\text { determine change on } \\
\text { SSAM or DOCS }\end{array}$ & Assessment time-points & $\begin{array}{l}\text { Other } \\
\text { outcome } \\
\text { measures at } \\
\text { concurrent } \\
\text { time-points }\end{array}$ & Findings \\
\hline & & $\begin{array}{l}\text { P4: Development of } \\
\text { rehabilitation goals } \\
\text { and pharmacological } \\
\text { intervention. }\end{array}$ & & & & $\begin{array}{l}\text { P3: new seizure } \\
\text { medication and } \\
\text { resultant stopping of } \\
\text { seizure activity via } \\
\text { EEG } \\
\text { P4:goal developed and } \\
\text { adjusted based on } \\
\text { abilities as measured } \\
\text { by the DOCS } \\
\text { (higher-level localised } \\
\text { responses elicited } \\
\text { with visual stimulation } \\
\text { - led to auditory } \\
\text { response goals) }\end{array}$ \\
\hline $\begin{array}{l}\text { Pape et al., } \\
2009 \\
\text { USA }\end{array}$ & $\begin{array}{l}N=113, \\
\text { TBI, } N=83 ; \text { OBI, } N= \\
30 \text { (OBI comprised } \\
\text { anoxia, cancer, } \\
\text { stroke, haemorrhage, } \\
\text { aneurysm and AVM) } \\
\text { GCS < } 8 \text { at enrolment } \\
\text { TPI at baseline } \mathrm{DOCS}: \\
\text { TBI: } 45.93 \text { days, } \mathrm{SD}= \\
20.9 \\
\text { OBI: } 55.1 \text { days, } \mathrm{SD}= \\
18.39 \\
\text { Sex: } 750^{7} / 38 \text { o } \\
\text { Age: } \mathrm{M}=38 \mathrm{SD}= \\
17.8 \text { years }\end{array}$ & $\begin{array}{l}\text { Inpatient rehabilitation. } \\
\text { Average length of } \\
\text { rehabilitation } \\
\text { hospitalisation }=59 \\
\text { days (SD }=36.38 \\
\text { days). }\end{array}$ & $\begin{array}{l}\text { Means and SD for } \\
\text { DOCS total at } \\
\text { baseline and } \\
\text { time-points 2-6 } \\
\text { reported for } \\
\text { participants evaluated } \\
\text { Baseline } \\
\text { Time 1: } N=113 \\
\text { Time 2: } N=92 \\
\text { Time 3: } N=63 \\
\text { Time 4: } N=45 \\
\text { Time 5: } N=32 \\
\text { Time 6: } N=22 \text {. }\end{array}$ & $\begin{array}{l}\text { Baseline DOCS (within } \\
144 \text { hours of } \\
\text { admission to } \\
\text { rehabilitation) then } \\
\text { repeated } \\
\text { approximately every } \\
7 \text { days on up to } 6 \\
\text { consecutive occasions }\end{array}$ & & $\begin{array}{l}\text { Change Baseline to time } \\
2 \text { for sample: } \\
(N=92) M=4.24, \\
S D=10.37 \\
\text { Change Baseline to time } \\
3 \text { for sample: } \\
(N=63) M=5.94 \\
S D=9.8 \\
\text { Change Baseline to time } \\
4 \text { for sample: } \\
(N=45) M=6.94, \\
S D=11.32 .\end{array}$ \\
\hline
\end{tabular}




\section{TABLE 4}

Continued.

\begin{tabular}{|c|c|c|c|c|c|c|}
\hline $\begin{array}{l}\text { Study (author, } \\
\text { date, country } \\
\text { of lead author) }\end{array}$ & $\begin{array}{l}\text { No. of participants/ } \\
\text { diagnosis/ injury } \\
\text { severity on admission to } \\
\text { study (ranges, means/ } \\
\text { VS/MCS/RLAS/GCS)/ } \\
\text { Time post-injury/ Sex } \\
\text { /Age (range, mean) }\end{array}$ & $\begin{array}{l}\text { Treatment description } \\
\text { (duration and intensity) }\end{array}$ & $\begin{array}{l}\text { Statistical method to } \\
\text { determine change on } \\
\text { SSAM or DOCS }\end{array}$ & Assessment time-points & $\begin{array}{l}\text { Other } \\
\text { outcome } \\
\text { measures at } \\
\text { concurrent } \\
\text { time-points }\end{array}$ & Findings \\
\hline & & & $\begin{array}{l}\text { Mean change scores } \\
\text { and SD tabulated for } \\
\text { change from baseline } \\
\text { to time } 2 \text {, baseline to } \\
\text { time } 3, \text { baseline to } \\
\text { time } 4 \text {, baseline to } \\
\text { time } 5 \text {, and baseline } \\
\text { to time } 6 \text {. } \\
\text { No statistical analysis of } \\
\text { change. }\end{array}$ & & & $\begin{array}{l}\text { Change Baseline to time } \\
5 \text { for sample: } \\
(N=32) M=10.10, \\
\text { SD }=14.50 \text {. } \\
\text { Change from Baseline to } \\
\text { time } 6 \text { for sample: } \\
(N=22) M=6.20, S D \\
=10.40 . \\
\text { The average amount of } \\
\text { DOCS score change } \\
\text { from baseline to each } \\
\text { subsequent DOCS } \\
\text { score increased from } \\
\text { Baseline to time } 5, \\
\text { with reduction at time } \\
6 .\end{array}$ \\
\hline
\end{tabular}

See the footnote to Table 1 for abbreviations. 
While the responsiveness of our measures to detect change in a statistical sense is important, this should always be assessed in conjunction with clinical judgement. Outcome measures of disorders of consciousness may not be representative of the amount of change which constitutes a clinically meaningful change for an individual. For example, a very slight improvement in one area on a consciousness scale may result in other changes that are meaningful to the person and their quality of life, but may not constitute statistically significant change overall. For example, an improvement in tactile responsiveness may enable increased interactions with family; improved object manipulation may enable some participation in tasks; or increased arousal and improved and consistent tracking may result in being able to make choices and communicate using a yes/no visual communication system. Determining clinically meaningful difference for consciousness measures poses great challenges because people in VS or MCS are unable to communicate what constitutes meaningful change, and meaningful change is different for each individual. Perhaps the answer to capturing rehabilitation outcomes with patients in MCS, where consciousness assessment is indicating slight but not statistically significant changes in response to treatment efforts, is to use individualised goal attainment to ensure that the targets of our treatment are captured. It is interesting to note that most of the studies reviewed used group statistical analysis of change. Furthermore, in the case of $n$-of- 1 studies or studies with small samples included in our review, descriptive statistics were used to analyse change rather than analysis of clinically or statistically significant change (i.e., reliable change index; Perdices, 2005).

The main limitations of this review are that the methodological quality of the included studies was not evaluated, because, to the authors' knowledge, there are no tools that specially evaluate the quality of studies designed to investigate sensitivity to change. However, included papers have been comprehensively summarised. Furthermore, this review was not a systematic review using rigorous methods characteristic of such a process, such as selection of included studies by two independent reviewers and strict inclusion and exclusion criteria tailored to a highly specific search question. Rather, a systematic search strategy was used to explore the available literature on responsiveness of the six measures of consciousness. The narrow search strategy utilised (i.e., a keyword search of databases using the full titles of the six measures) also limited the scope of the literature search, and further available studies may have been identified if the search strategy had been broader. However, to counteract this, the reference lists on included studies were checked to identify any previous related studies. There are also numerous other measures of consciousness available that were not the subject of this review and may be useful for monitoring change. Some of these measures include the C/NCS (Rappaport et al., 1992), the Comprehensive Levels of Consciousness Scale (Stanczak et al., 1984), the Full Outline of Unresponsiveness Scale (Wijdicks, Bamlet, Maramattom, Manno, \& McClelland, 2005), the Innsbruck Coma Scale (Benzer et al., 1991), the Loewenstein Communication Scale (Borer-Alafi, Gil, Sazbon, \& Korn, 2002), the Swedish Reaction Level Scale- 1985 (Stalhammar et al., 1988) and the Glasgow-Liege Coma Scale (Born, Albert, Hans, \& Bonnal, 1985).

Due to the lack of consensus on the most ideal statistical method for determining responsiveness of a measure, future studies should perhaps seek to examine responsiveness using a range of available methods. Furthermore, a wider retrospective examination of responsiveness could have been possible; however, many studies did not report the required data (i.e., means, standard deviations and/or raw scores for case studies or small samples at different time points). Treatment studies in this area are faced with the challenge of the rare condition and subsequently, small sample sizes. However, if such data are reported, it would enable data to be pooled to conduct meta-analyses. To enable between-study comparisons and pooling of data, future studies would also need to include information about: measurement time-points; treatment content, intensity and duration; and thorough participant information, including injury severity and time post-injury.

\section{Conclusions}

The findings of this review indicate that there is some evidence for the ability of the six measures to detect change following treatment. The CRS-R, WNSSP and WHIM have established responsiveness to change, given that several studies have incorporated statistical analysis to demonstrate significant change and there was at least one study where Cohen's $d$ was able to be determined, demonstrating large effect sizes for each of these measures. The SMART has some evidence of responsiveness, based on one study demonstrating statistically significant change with a large effect size, as does the SSAM, based on one study incorporating statistical analysis of change only. The DOCS has preliminary evidence for responsiveness based on one study using descriptive analysis of change data over time without statistical analysis of change. Future studies should seek to 
examine responsiveness to further establish the utility of available measures of consciousness to reliably measure change in response to treatment.

\section{References}

Ansell, B.J., \& Keenan, J.E. (1989). The Western Neuro Sensory Stimulation Profile: a tool for assessing slow-to-recover head-injured patients. Archives of Physical Medicine and Rehabilitation, 70(2), 104108.

Bekinschtein, T.A., Tiberti, C., Niklison, J., Tamashiro, M., Ron, M., Carpintiero, S., ... Manes, F. (2005). Assessing level of consciousness and cognitive changes from vegetative state to full recovery. $\mathrm{Neu}$ ropsychological Rehabilitation, 15(3-4), 307-322.

Benzer, A., Mitterschiffthaler, G., Marosi, M., Luef, G., Pühringer, F., De La Renotiere, K., ... Schmutzhard, E. (1991). Prediction of non-survival after trauma: Innsbruck Coma Scale. Lancet, 338(8773), 977-978.

Borer-Alafi, N., Gil, M., Sazbon, L., \& Korn, C. (2002). Loewenstein communication scale for the minimally responsive patient. Brain Injury, 16(7), 593-609.

Born, J.D., Albert, A., Hans, P., \& Bonnal, J. (1985). Relative prognostic value of best motor response and brain stem reflexes in patients with severe head injury. Neurosurgery, 16(5), 595-601.

Cohen, J. (1988). Statistical power analysis for the behaviour sciences (2nd ed.). Hillsdale NJ: Lawrence Erlbaum Associates.

Crossley, M., Shiel, A., Wilson, B., Coleman, M.R., Geling, L., Fryer, T., ... Pickard, J. (2005). Monitoring emergence from coma following severe brain injury in an octogenarian using behavioural indicators, electrophysiological measures and metabolic studies: a demonstration of the potential for good recovery in older adults. Brain Injury, 19(9), 729-737.

Cruse, D., Chennu, S., Chatelle, C., Fernandez-Espejo, D., Bekinschtein, T.A., Pickard, J.D., ... Owen, A.M. (2012). Relationship between etiology and covert cognition in the minimally conscious state. Neurology, 78, 816-822.

Davis, A.E., \& Gimenez, A. (2003). Cognitivebehavioural recovery in comatose patients following auditory sensory stimulation. Journal of Neuroscience Nursing, 35(4), 202-209.

DeFina, P.A., Fellus, J., Thompson, J.W.G., Eller, M., Moser, R.S., Frisina, P.G., ... Prestigiacoma, C.J. (2010). Improving outcomes of severe disorders of consciousness. Restorative Neurology and Neuroscience, 28(6), 769-780.

Eilander, H.J., van de Wiel, M., Wijers, M., van Heugten, C.M., Buljevac, D., Lavrijsen, J.C.M., ... Prevo, A.J.H. (2009). The reliability and validity of the PALOCS: a post-acute level of consciousness scale for assessment of young patients with prolonged disturbed consciousness after brain injury. Neuropsychological Rehabilitation, 19(1), 1-27.
Elliot, L., Coleman, M., Shiel, A., Wilson, B.A., Badwan, D., Menon, D., ... Pickard, J. (2005). Effect of posture on levels of arousal and awareness in vegetative and minimally conscious patients: a preliminary investigation. Journal of Neurology, Neurosurgery and Psychiatry, 76(2), 298-299.

Giacino, J.T., Ashwal, S.A., Childs, N., Cranford, R., Jennett, B., Katz, D.I., ... Zasler, N. (2002). The minimally conscious state: definition and diagnostic criteria. Neurology, 58, 349-353.

Giacino, J.T., \& Kalmar, K. (1997). The vegetative and minimally conscious states: a comparison of clinical features and functional outcome. Journal of Head Trauma Rehabilitation, 12(4), 36-51.

Giacino, J.T., Kalmar, K., \& Whyte, J. (2004). The JFK Coma Recovery Scale - Revised: Measurement characteristics and diagnostic utility. Archives of Physical Medicine and Rehabilitation, 85(12), 20202029.

Giacino, J.T., Zasler, N.D., Katz, D.I., Kelly, J.P., Rosenberg, J.H., \& Filley, C.M. (1997). Development of practice guidelines of assessment and management of the vegetative and minimally conscious states. Journal of Head Trauma Rehabilitation,12(4), 7989.

Gill-Thwaites, H., \& Munday, R. (2004). The sensory modality assessment and rehabilitation technique (SMART): a valid and reliable assessment for vegetative state and minimally conscious state patients. Brain Injury, 18(12), 1255-1269.

Guyatt, G.H., Deyo, R.A., Charlson, M., Levine, M.N., \& Mitchell, A. (1989). Responsiveness and validity in health status measurement: a clarification. Journal of Clinical Epidemiology, 42(5), 403-408.

Hall, M.E., MacDonald, S., \& Young, G.C. (1992). The effectiveness of directed multisensory stimulation versus non-directed stimulation in comatose $\mathrm{CHI}$ patients: pilot study of a single subject design. Brain Injury, 6(5), 435-445.

Husted, J.A., Cook, R.J., Farewell, V.T., \& Gladman, D.D. (2000). Methods for assessing responsiveness: a critical review and recommendations. Journal of Clinical Epidemiology, 53(5), 459-468.

Jennett, B., \& Plum, F. (1972). Persistent vegetative state after brain damage: a syndrome in search of a name. Lancet, 1(7753), 734-737.

Katz, D.I., Polyak, M., Coughlan, D., Nicols, M., \& Roche, A. (2009). Natural history of recovery from brain injury after prolonged disorders of consciousness: outcome of patients admitted to inpatient rehabilitation with 1-4 year follow-up. Progress in Brain Research, 177, 73-88.

Kazis, L.E., Anderson, J.J., \& Meenan, R.F. (1989). Effect sizes for interpreting changes in health status. Medical Care, 27(3), S178-S189.

Lammi, M.H., Smith, V.H., Tate, R.L., \& Taylor, C.M. (2005). The minimally conscious state and recovery potential: a follow-up study 2 to 5 years after traumatic brain injury. Archives of Physical Medicine and Rehabilitation, 86, 746-754. 
Lancioni, G.E., O’Reilly, M.F., Singh, N.N., Buonocunto, F., Sacco, V., Colonna, F., ... Bosco, A. (2009). Technology-based intervention options for post-coma persons with minimally conscious state and pervasive motor disabilities. Developmental Neurorehabilitation, 12(1), 24-31.

Lancioni, G., Singh, N., O’Reilly, M., Olivetti, M., De Tommaso, M., Navarro, J., ... Sacco, V. (2011). A learning assessment procedure as a test supplement for monitoring progress with two post-coma persons with a diagnosis of vegetative state. Developmental Neurorehabilitation, 14(6), 358-365.

Majerus, S., Gill-Thwaites, H., Andrews, K., \& Laureys, S. (2005). Behavioural evaluation of consciousness in severe brain damage. Progress in Brain Research, 150, 397-413.

Majerus, S., \& Van de Linden, M. (2000). Wessex head injury matrix and Glasgow/Glasgow-Liege coma scale: a validation and comparison study. Neuropsychological Rehabilitation, 10(2), 167-184.

McMahon, M.A., Vargus-Adams, J.N., Michaud, L.J., \& Bean, J. (2009). Effects of amantadine in children with impaired consciousness caused by brain injury. American Journal of Physical Medicine and Rehabilitation, 88, 525-532.

Noé, E., Olaya, J., Navarro, M.D., Noguera, P., Colomer, C., Garcia-Panach, J., ... Ferri, J. (2012). Behavioural recovery in disorders of consciousness: a prospective study with the Spanish version of the coma recovery scale-revised. Archives of Physical Medicine and Rehabilitation, 93, 428-433.

Olejnik, S., \& Algina, J. (2000). Measure of the effect size for comparative studies: applications, interpretations and limitations. Contemporary Educational Psychology, 25, 241-286.

Pape, T.L.-R., Heinemann, A.W., Kelly, J.P., Hurder, A.G., \& Lundgren, S. (2005a). A measure of neurobehavioural functioning after coma - Part 1: Theory, reliability and validity of the disorders of consciousness scale. Journal of Rehabilitation Research and Development, 42(1), 1-18.

Pape, T.L.-B., Senno, R.G., Guernon, A., \& Kelly, J.P. (2005b). A measure of neurobehavioural functioning after coma. Part II: Clinical and scientific implementation. Journal of Rehabilitation Research and Development, 42(1), 19-28.

Pape, T.L.-B., Tang, C., Guernon, A., Lundgren, S., Blahnik, M., Yongliang, W., ... Soneru, I. (2009). Predictive value of the disorders of consciousness scale (DOCS). Physical Medicine and Rehabilitation, 1(2), 152-161.

Patrick, P.D., Wamstad, J.B., Mabry, J.L., Smith-Janik, S., Gurka, M.J., Buck, M.L., ... Blackman, J.A. (2009). Assessing the relationship between the WNSSP and therapeutic participation in adolescents in low response states following severe traumatic brain injury. Brain Injury, 23(6), 528-534.

Perdices, M. (2005). How do you know your patient is getting better (or worse?) A user's guide. Brain Impairment, 6(3), 219-226.
Piccione, F., Cavinato, M., Manganotti, P., Formaggio, E., Storti, S.F., Battistin, L., ... Dam, M. (2011). Behavioural and neuropsychological effects of repetitive transcranial magnetic stimulation on the minimally conscious state: a case study. Neurorehabilitation and Neural Repair, 25(1), 98-102.

Portney, L.G., \& Watkins, M.P. (2000). Foundations of clinical research: Applications to practice (2nd ed.). New Jersey: Prentice Hall Health.

Rader, M.A., Alston, J.B., \& Ellis, D.W. (1989). Sensory stimulation of severely brain injured patients. Brain Injury, 3, 141-147.

Rader, M.A., \& Ellis, D.W. (1994). The Sensory Stimulation Assessment Measure (SSAM): a tool for early evaluation of severely brain injured patients. Brain Injury, 8, 309-321.

Rappaport, M., Dougherty, A.M., \& Kelting, D.L. (1992). Evaluation of coma and vegetative states. Archives of Physical Medicine and Rehabilitation, 73(7), 628634.

Rappaport, M., Hall, K.M., Hopkins, K., Belleza, T., \& Cope, D.N. (1982). Disability rating scale for severe head trauma: coma to community. Archives of Physical and Medical Rehabilitation, 63(3), 118-123.

Rodriguez Moreno, D., Schiff, N.D., Giacino, J., Kalmar, K., \& Hirsch, J. (2010). A network approach to assessing cognition in disorders of consciousness. Neurology, 75(21), 1871-1878.

Royal College of Physicians. (2003). Rehabilitation following acquired brain injury - national clinical guidelines. London: Lavenham Press.

Sara, M., Pistoia, F., Mura, E., Onorati, P., \& Govoni, S. (2009). Intrathecal baclofen in patients with persistent vegetative state: 2 hypotheses. Archives of Physical Medicine and Rehabilitation, 90, 12451249.

Schnakers, C., Hustinx, R., Vandewalle, G., Majerus, S., Moonen, G., Boly, M., . . . Laureys, S. (2008). Measuring the effect of amantadine in chronic anoxic minimally conscious state. Journal of Neurology, Neurosurgery and Psychiatry, 79, 225-227.

Seel, R.T., Sherer, M., Whyte, J., Katz, D.I., Giacino, J.T., Rosenbaum, A.M., ... Zasler, N. (2010). Assessment scales for disorders of consciousness: evidence-based recommendations for clinical practice and research. Archives of Physical Medicine and Rehabilitation, 91, 1795-1813.

Shiel, A., Horn, S., Wilson, B., Watson, M., Campbell, M., \& McLellan, L. (2000a). The Wessex Head Injury Matrix (WHIM) main scale: a preliminary report on a scale to assess and monitor patient recovery after severe head injury. Clinical Rehabilitation, 14(4), 408-416.

Shiel, A., Wilson, B.A., McLellan, D.L., Horn, S.A., \& Watson, M. (2000b). The Wessex Head Injury Matrix (WHIM). Bury St Edmunds, England: Thames Valley Test Company.

Smith, V.H., Taylor, C.M., Lammi, M.H., \& Tate, R.L. (2001). Recovery profiles of cognitive-sensory modalities in patients in the minimally conscious 
state following traumatic brain injury. Brain Impairment, 2(1), 29-38.

Stalhammar, D., Starmark, J.E., Holmgren, E., Eriksson, N., Nordstrom, C.H., Fedders, O., \& Rosander, B. (1988). Assessment of responsiveness in acute cerebral disorders: a multicentre study on the reaction level scale (RLS 85). Acta Neurochirurgica, 90(34), 73-80.

Stanczak, D.E., White, J.G. 3rd, Gouview, W.D., Moehle, K.A., Daniel, M., Novack, T., ... Long, C.J. (1984). Assessment of level of consciousness following severe neurological insult: a comparison of the psychometric qualities of the Glasgow Coma Scale and the Comprehensive Level of Consciousness Scale. Journal of Neurosurgery, 60(5), 955-960.

The Multi-Society Task Force on PVS. (1994). Medical aspects of persistent vegetative state. The New England Journal of Medicine, 330(21), 1499-1508.

Vargus-Adams, J.N., McMahon, M.A., Michaud, L., Bean, J., \& Vinks, A.A. (2010). Pharmacokinetics of amantadine in children with impaired consciousness due to acquired brain injury: preliminary findings using a sparse-sampling technique. Physical Medicine and Rehabilitation, 2, 37-42.

Whyte, J., \& DiPasquale, M.C. (1995). Assessment of vision and visual attention in minimally responsive brain injured patients. Archives of Physical Medicine and Rehabilitation, 76(9), 804-810.

Whyte, J., DiPasquale, M.C., \& Vaccaro, M. (1999). Assessment of command-following in minimally conscious brain injured patients. Archives of Physical Medicine and Rehabilitation, 80(6), 653-660.

Whyte, J., \& Myers, R. (2009). Incidence of clinically significant responses to Zolpidem among patients with disorders of consciousness. American Journal of Physical Medicine and Rehabilitation, 88(5), 410418.

Wijdicks, E.F., Bamlet, W.R., Maramattom, B.V., Manno, E.M., \& McClelland, R.L. (2005). Validation of a new coma scale: the FOUR score. Annals of Neurology, 58(4), 585-593.

Wilson, B.A., Coleman, M.R., \& Pickard, J.D. (2008). Neuropsychological assessment and management of people in states of impaired consciousness: an overview of some recent studies. Brain Impairment, 9(1), 28-35.

Wilson, C.F., Elder, V., McCrudden, E., \& Caldwell, S. (2009). Analysis of Wessex Head Injury Matrix (WHIM) scores in consecutive vegetative and minimally conscious state patients. Neuropsychological Rehabilitation, 19(5), 754-760.

Wilson, F.C., Harpur, J., Watson, T., \& Morrow, J I. (2003). Adult survivors of severe cerebral hypoxia - case series survey and comparative analysis. $\mathrm{Neu}$ rorehabilitation, 18, 291-298.

Wilson, S.L., \& Gill-Thwaites, H. (2000). Early indication of emergence from vegetative state derived from assessments with the SMART - a preliminary report. Brain Injury, 14(4), 319-331.

Wright, J.G., \& Young, N.L. (1997). A comparison of different indices of responsiveness. Journal of Clinical Epidemiology, 50(3), 239-246.

Zakzanis, K.K. (2001). Statistics to tell the truth, the whole truth, and nothing but the truth: formulae, illustrative numerical examples, and heuristic interpretation of effect size analyses for neuropsychological researchers. Archives of Clinical Neuropsychology, 16(16), 653-667. 